Subscriber access provided by King Abdullah University of Science and Technology Library

\title{
Article
}

\section{Coalescence dynamics of mobile and immobile fluid interfaces}

Ivan U Vakarelski, Rogerio Manica, Er Qiang Li, Elka S

Basheva, Derek Y. C. Chan, and Sigurdur T. Thoroddsen

Langmuir, Just Accepted Manuscript • DOI: 10.1021/acs.langmuir.7b04106 • Publication Date (Web): 12 Jan 2018

Downloaded from http://pubs.acs.org on January 18, 2018

\section{Just Accepted}

"Just Accepted" manuscripts have been peer-reviewed and accepted for publication. They are posted online prior to technical editing, formatting for publication and author proofing. The American Chemical Society provides "Just Accepted" as a free service to the research community to expedite the dissemination of scientific material as soon as possible after acceptance. "Just Accepted" manuscripts appear in full in PDF format accompanied by an HTML abstract. "Just Accepted" manuscripts have been fully peer reviewed, but should not be considered the official version of record. They are accessible to all readers and citable by the Digital Object Identifier (DOI®). "Just Accepted" is an optional service offered to authors. Therefore, the "Just Accepted" Web site may not include all articles that will be published in the journal. After a manuscript is technically edited and formatted, it will be removed from the "Just Accepted" Web site and published as an ASAP article. Note that technical editing may introduce minor changes to the manuscript text and/or graphics which could affect content, and all legal disclaimers and ethical guidelines that apply to the journal pertain. ACS cannot be held responsible for errors or consequences arising from the use of information contained in these "Just Accepted" manuscripts. 


\title{
Coalescence dynamics of mobile and immobile fluid
}

\section{interfaces}

\author{
Ivan U. Vakarelski, ${ }^{1 *}$ Rogerio Manica, ${ }^{2}$ Er Qiang Li, ${ }^{1,3}$ Elka S. Basheva, ${ }^{4}$ Derek Y. C. Chan ${ }^{5,6}$ \\ and Sigurdur T. Thoroddsen ${ }^{1}$
}

${ }^{1}$ Division of Physical Sciences and Engineering, King Abdullah University of Science and Technology (KAUST), Thuwal, 23955-6900, Saudi Arabia

${ }^{2}$ Department of Chemical and Materials Engineering, University of Alberta, Edmonton, Alberta, T6G 1H9, Canada

${ }^{3}$ Department of Modern Mechanics, University of Science and Technology of China, Hefei 230027, China

${ }^{4}$ Department of Chemical and Pharmaceutical Engineering, Faculty of Chemistry and Pharmacy, Sofia University, Sofia, Bulgaria

${ }^{5}$ School of Mathematics and Statistics, University of Melbourne, Parkville, VIC 3010, Australia.

${ }^{6}$ Department of Mathematics, Swinburne University of Technology, Hawthorn, VIC 3122, Australia.

* CORRESPONDING AUTHOR:

Ivan U. Vakarelski, E-mail: ivanuriev.vakarelski@kaust.edu.sa

KEYWORDS: Bubbles, Droplets, Surface mobility, Coalescence 


\begin{abstract}
Coalescence dynamics between deformable bubbles and droplets can be dramatically affected by the mobility of the interfaces with fully tangentially mobile bubble-liquid or droplet-liquid interfaces expected to accelerate the coalescence by orders of magnitudes. However, there is a lack of systematic experimental investigations that quantify this effect. By using high speed camera imaging we examine the free rise and coalescence of small air-bubbles (100 to $1300 \mu \mathrm{m}$ in diameter) with a liquid interface. A perfluorocarbon liquid, PP11 is used as a model liquid to investigate coalescence dynamics between fully-mobile and immobile deformable interfaces. The mobility of the bubble surface was determined by measuring the terminal rise velocity of small bubbles rising at Reynolds numbers, Re less than 0.1 and the mobility of free PP11 surface by measuring the deceleration kinetics of the small bubble toward the interface. Induction or film drainage times of a bubble at the mobile PP11-air surface were found to be more than two orders of magnitude shorter compared to the case of bubble and an immobile PP11-water interface. A theoretical model is used to illustrate the effect of hydrodynamics and interfacial mobility on the induction time or film drainage time. The results of this study are expected to stimulate the development of a comprehensive theoretical model for coalescence dynamics between two fully or partially mobile fluid interfaces.
\end{abstract}




\section{INTRODUCTION}

The mechanisms that control the speed of coalescence between gas bubbles and emulsion droplets are of continual interest due to their relevance to a wide range of practical applications, from the optimizing of processing of food and pharmaceutical products to the efficient recovery of crude oil. More generally the science of coalescence of soft deformable bodies also underpins many assembly and dynamic processes that occur in material science and biology. As bubbles and droplets approach, the hydrodynamic interaction is the initially dominant interaction at large separations that can cause local deformation that subsequently determine the effectiveness of shorter ranged forces such as electrical double layer, van der Waals, steric and molecularly specific forces that will eventually operate to dictate the outcome of the coalescence process. ${ }^{1-3}$

The important physical attribute that controls the magnitude and characteristic time scale of hydrodynamic interactions between deformable bubbles or droplets is their surface mobility. The accepted paradigm is that fluid molecules adjacent to a solid surface will move with the same velocity as the surface in both the normal and tangential directions. This is often referred to as the no-slip or stick boundary condition. Here, we will refer to this as an immobile interface or an immobile hydrodynamic boundary condition. In contrast, it is assumed that a clean gas-liquid interface cannot sustain any shear stress and this is referred to as a free-slip or a fully mobile interface. The liquid-liquid interface between two immiscible phases represents the intermediate case of a partially mobile interface, the mobility of which depends on the viscosity ratio of the two liquids. Thus a drop with a viscosity very much larger than the surrounding fluid will behave like a solid. In practice, a fully mobile interface may be hard to realize because small amounts of surface active impurities or additives that are usually present in liquids can lead to partial or even 
full immobilization of the bubble or droplet interface whereby the hydrodynamic boundary condition resembles the immobile condition at a liquid-solid interface. Moreover, the complex hydrodynamic condition at a solid surface that possesses small scale geometric structures or has been treated with a thin coating of adsorbates or has adsorbed gas bubbles, it is often subsumed in the notion of a partially mobile surface characterized by a phenomenological slip length. ${ }^{4}$

However, since a fully mobile interface cannot sustain any shear stress, it is expected that the rate of coalescence of two bubbles or drops with viscosities much smaller than the surrounding fluid should be much faster than in cases where an immobile surface is involved because of the much lower viscous resistance at mobile interfaces to the drainage and thinning of the intervening fluid film. However at present, due to possible deformation of the interface that may occur over different length scales, theoretical modelling of the coalescence dynamics of two bubbles or drops with mobile interfaces either involved demanding numerical computations, ${ }^{5}$ making it difficult to expose the key physics, or in simpler models involved questionable assumptions as the use of the lubrication approximation to describe film drainage between two fully mobile interfaces ${ }^{6}$ which could not be validated as it leads to a slowly divergent pressure profile. $^{1}$

Although a clean gas-liquid interface is an obvious model of a mobile interface, experimental observation of coalescence dynamics between deformable fully-mobile interfaces has significant challenges. The interface of the most practically relevant liquid, water is notorious for being easily immobilized by even trace amounts of impurities. For instance, in the study of the terminal rise velocities of small bubbles in water that are below $100 \mu \mathrm{m}$ in diameter whereby the hydrodynamics is in the Stokes flow or low Reynolds number $(\operatorname{Re}<1)$ regime with viscous forces dominant over inertial forces, extreme effort was required to purify the water in 
order to maintain a fully-mobile bubble-water interface to furnish results that agree with theoretical predictions. ${ }^{7,8}$ Similar stringent cleanliness for ultra-pure water is also required in the study of larger bubbles of diameter up to $600 \mu \mathrm{m}$, for which the bubble trajectories remain rectilinear and the rise velocity reaches a characteristic maximum of around $32 \mathrm{~cm} / \mathrm{s}$ for agreement between experimental observations and theoretical predictions based on freely mobile bubbles. ${ }^{9}$ Larger size bubbles exhibit spiral or zig-zag trajectories. It has been shown for bubbles at a size near the velocity maximum $(\operatorname{Re} \sim 600)$, the presence of impurities at the trace concentrations $\left(\sim 10^{-5} \mathrm{M}\right)$ will reduce the terminal rise velocity to that resembling an immobile bubble interface, like that of a solid surface. ${ }^{10}$

The collision of mobile bubbles in ultra-pure water at the size corresponding to the maximum rise velocity has been studied at mica, Teflon and water-air interfaces. ${ }^{10-16}$ The collision dynamics of rising bubbles in Millipore water that have immobile interfaces at a glass surface have also been studied. ${ }^{17}$ The multiple rebounds observed in such collisions can be explained in terms of hydrodynamic interactions and the restoring forces due to bubble deformation. ${ }^{18}$ In fact, being in close proximity to a solid surface can lead to immobilization of the bubble interface due to impurities originating from the solid. ${ }^{19,20}$ In other experiments when a mobile interface bubble rises toward the free water surface the bubble bounce and coalescence kinetics indicate that even in the case of ultrapure water, the free water surface appears to be immobile, probably due to contamination that originated from the laboratory atmosphere, and similar results have also been obtained with other polar liquids as ethanol. ${ }^{21}$

From an experimental perspective, comprehensive explorations of the effects of varying surface mobility using well characterized and reproducible systems that allow quantitative comparisons are still lacking. Coalescence studies involving bubbles as well as hydrocarbon and 
fluorocarbon droplets in water, all below the $100 \mu \mathrm{m}$ size range, have been conducted using the atomic force microscope (AFM). ${ }^{1,22-24}$ By mounting bubbles or droplets on the AFM microcantilever the interaction force can be measured as a function of time as the bubbles or droplets are driven together or pulled apart in controlled ways. In all cases, good quantitative agreement was obtained with theories that assumed the immobile hydrodynamic boundary condition on the deformable interfaces. Experiments that use macroscopic size bubbles ${ }^{25,26}$ or mercury drops ${ }^{27}$ in water also indicated the immobile boundary condition is applicable. However, flow induced coalescence of small polybutadiene drops $(<100 \mu \mathrm{m})$ suspended in polydimethylsiloxane where both phases have viscosities 3 to 5 orders of magnitudes higher then water, demonstrated the viscosity ratio effect on interfacial mobility and coalescence. ${ }^{5,28}$ Experiment on the film drainage between a gas bubble and a free surface in very high viscosity liquid as glass melts agree as well with mobile surface drainage model. ${ }^{29,30}$ Under certain conditions the rapid drainage dynamics of thin water films observed in a capillary cell was attributed to surface mobility. ${ }^{31-33}$ Recently, it has been shown that the high-speed entry of a superhydrophobic sphere into water or of a hot metallic sphere in the Leidenfrost state into liquid can entrain a giant gas cavity that is around 15 times the size of the sphere. ${ }^{34}$ The surface of this cavity is shown to be fully mobile and is responsible for the reduction of the hydrodynamic drag by about $90 \%$. Thus there remain novel phenomena associated with fully mobile interfaces. Nonetheless, there is a lack of systematic observations that clearly contrast the coalescence dynamics of bubbles or drops with mobile and with immobile interfaces.

More importantly, in modelling the flow of water adjacent to a solid hydrophobic surface, it is often assumed that a partial slip hydrodynamic boundary condition applies, with a slip length that depends on the degree of hydrophobicity of the surface. ${ }^{35}$ This simple model subsumed any 
small scale local structural details and/or the presence of adsorbates on hydrophobized surfaces into a single parameter. Consequently, the phenomenon of "hydrodynamic slip boundary condition" and "surface hydrophobicity" is often taken to be co-existing and synonymous even though there is little direct experimental evidence to support their association. The present work will test the veracity of this apparent link between hydrophobic or hydrophobized surfaces and hydrodynamic slip.

\section{PRESENT WORK}

To quantify the coalescence dynamics between two mobile deformable interfaces and to contrast that with cases where at least one immobile interface is involved, we conduct bubble rise experiments using a fluorocarbon liquid, Flutec PP11 (perfluoroperhydrophenanthrene $\left(\mathrm{C}_{14} \mathrm{~F}_{24}\right.$, F2 Chemical Ltd.) and observe the coalescence of the bubble with a flat liquid PP11-air or PP11water interface.

Perfluorocarbon liquids are chemically inert, completely immiscible with other liquids, including polar liquids such as water, as well as hydrocarbon oils. The free interfaces of such liquids are highly resistant to surface active contamination. They are therefore molecularly smooth hydrophobic surfaces. However, common gases (e.g. $\mathrm{O}_{2}$ and $\mathrm{N}_{2}$ ) are very soluble in perfluorocarbon liquids. The perfluorocarbon liquid PP11 chosen for this study has density, $\rho=$ $2.03 \mathrm{~g} / \mathrm{cm}^{3}$ and a higher shear viscosity, $\mu=19.2 \mathrm{mPa} \mathrm{s}$, compared to that for water, $\mu=1.0 \mathrm{mPa}$ s. This makes it easier to conduct experiments at lower speeds or small Reynolds numbers so as to give laminar flow conditions and also reduced deviations of the bubble shapes from spherical as they rise. On the other hand, the viscosity is low enough to facilitate exploration of the transition to higher Reynolds numbers that may be relevant for many practical applications. 
No less important is that the refractive index of the PP11 is identical to that of water in the optical frequency range, making it a good proxy for the van der Waals interaction of water. This fact has been exploited to greatly reduce the magnitude of the repulsive van der Waals disjoining pressure that prevented the coalescence of a bubble with a fluorocarbon-water interface where a perfluorocarbon liquid with lower refractive index than that of water was used. $^{36}$

To create immobile interfaces, we use water drops that contain a perfluorinated surfactant that adsorbs at the water-PP11 interface. The bubble and water drop sizes can also be varied to adjust the range of rise velocities.

\section{EXPERIMENTAL}

The perfluorocarbon liquid FLUTEC ${ }^{\odot}$ PP11, High Performance fluid from F2 Chemicals Ltd., used in the experiments was mostly composed of perfluoroperhydrophenanthrene $\left(\mathrm{C}_{14} \mathrm{~F}_{24}\right)$. The PP11 liquid is clear and colorless with refractive index, $n=1.33$ and density, $\rho=2.03 \mathrm{~g} / \mathrm{cm}^{3}$. The PP11 dynamic viscosity as measured with an Ubbelohde capillary viscometer was found to be, $\mu=19.2 \mathrm{mPa} \mathrm{s}$, a value that is about $60 \%$ of the nominal value specified by the manufacturer. ${ }^{37}$ The viscosity measurements and all related experiments were conducted at the laboratory temperature of about $23^{\circ} \mathrm{C}$.

Schematics of the experimental setup used to monitor the bubbles free rise and collision with the liquid surface are given in Figure 1. A glass container (cross section $2.5 \times 2.5 \mathrm{~cm}$, height 7.5 $\mathrm{cm}$ ) is partly filled with the PP11 liquid. Usually we filled about $5 \mathrm{~cm}$ of the glass container height with the PP11 liquid, and added about $1 \mathrm{~cm}$ of water on top of it in the case of PP11-water interface experiments. Bubbles are released from the fine end of a glass capillary mounted close 
to the bottom of the container. The fine end of the bubble release capillary with inner diameter 2 $\mu \mathrm{m}$ to $5 \mu \mathrm{m}$ was fabricated using a glass-puller (Shutter Instruments Ltd.) that heated and pulled an original glass capillary of 50 to $100 \mu \mathrm{m}$ inner diameter. The other end of the capillary is connected by a plastic tube to a pressure regulator (Iwashita Instruments Ltd.) used to generate controlled air flow pulses. Using combinations of different capillary fine end diameters and pressure pulse duration we were able to release air bubbles with diameters in the range of $50 \mu \mathrm{m}$ to $1400 \mu \mathrm{m}$. To create water drops in PP11, the fine-end capillary was connected by plastic tubing to a $10 \mathrm{ml}$ syringe filled with water solution, allowing the release of water droplets of diameters in the range of $80 \mu \mathrm{m}$ to $300 \mu \mathrm{m}$ that rise due to the buoyancy force.

The top free surface provides a flat PP11-air or a flat PP11-water interface, if water is added to create an upper phase above the PP11. The upper water phase was always an aqueous solution of $0.5 \mathrm{M} \mathrm{NaCl}$ at $\mathrm{pH}=3.0$ adjusted by the addition of $\mathrm{HCl}$. The use of high electrolyte concentration and low $\mathrm{pH}$ was aimed to minimize the water-oil interfacial surface charge. ${ }^{38}$

The bubble or drop free rise and collision with the top surface was recorded using a highspeed camera (Photron-SA3) equipped with a long-distance microscope tube with a $5 \times$ or $10 \times$ magnification objective (Mitutoyo), giving a resolution of $3.4 \mu \mathrm{m} /$ pixel or $1.6 \mu \mathrm{m} / \mathrm{pixel}$. The high-speed videos were taken using a typical rate of 1000 frames per second (fps) and shutter speed of 1/5000 s. Variations of the bubble coordinates and instantaneous velocity with time were determined by image processing the videos either by using the Photron software or a MATLAB image processing code. When processing videos of the bubble approach to the surface, a fifth-order polynomial function was usually employed to smooth the coordinate $v s$ time data. 


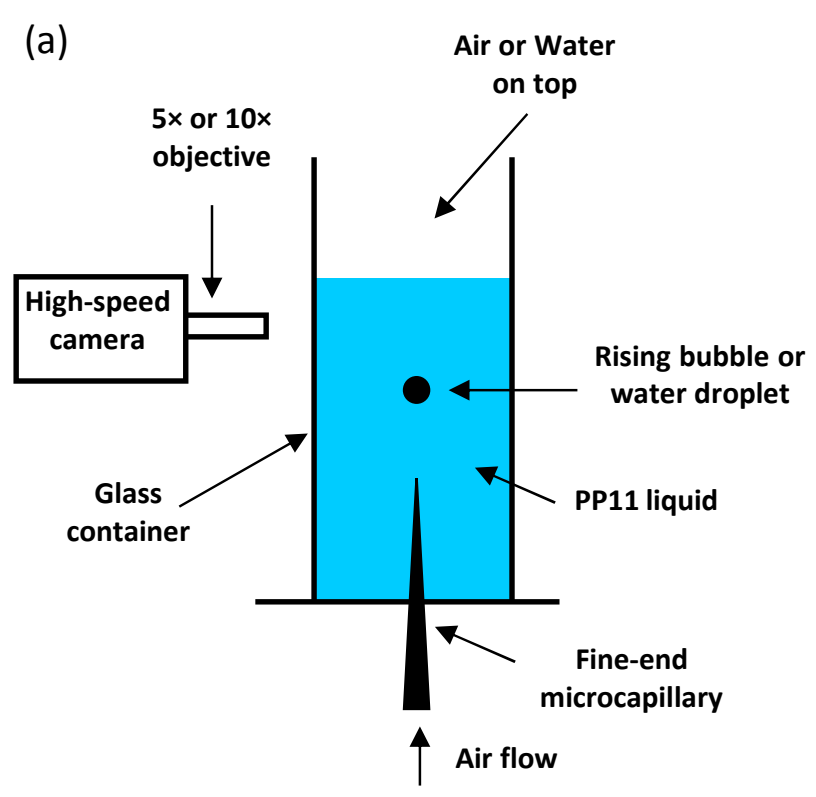

(b)

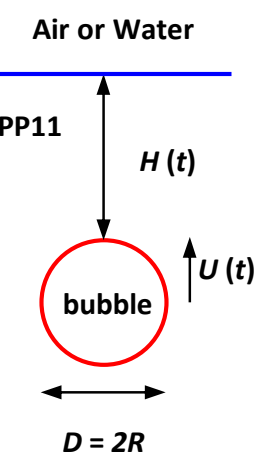

(c)

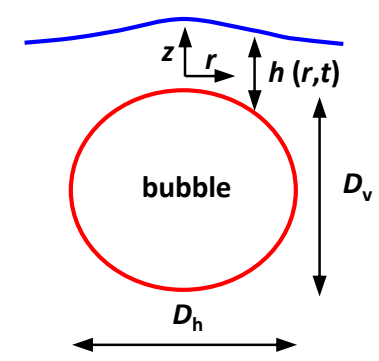

Figure 1. (a) Schematic of the experimental setup. (b) Schematic of a spherical bubble/droplet rising toward the flat PP11-air or PP11-water interface. (c) Schematic of the bubble impacting the deformable surface and forming an axisymmetric thin liquid film with film thickness $h(r, t)$. The film thickness shown in Fig. 9 is $h(r=0, t)$, the thickness at $r=0$.

In the terminal rise velocity experiments of water drops we used either Millipore purified water or Millipore water with added 0.1 wt $\%$ of the Nonionic Fluorosurfactant Zonyl® FSN $\left(\mathrm{F}\left(\mathrm{CF}_{2} \mathrm{CF}_{2}\right)_{3-8} \mathrm{CH}_{2} \mathrm{CH}_{2} \mathrm{O}\left(\mathrm{CH}_{2} \mathrm{CH}_{2} \mathrm{O}\right)_{\mathrm{x}} \mathrm{H}\right) .{ }^{39} \mathrm{~A}$ Krüss tensiometer was used to determine various surface and interfacial tensions: PP11-air $(21.5 \mathrm{mN} / \mathrm{m})$, PP11-water $(47.0 \mathrm{mN} / \mathrm{m})$ and PP11water with $0.1 \mathrm{wt} \%$ Zonyl FSN $(5.6 \mathrm{mN} / \mathrm{m})$, indicative of the adsorption of the fluorinated 
surfactant at the PP11-water interface. All experiments were conducted at room temperature of $23{ }^{\circ} \mathrm{C}$.

The side observation of the bubble/droplet approach to the surface does not allow precise determination of the separation $H$ and the film thickness, $h(r, t)$ when separation is much smaller than the bubble/droplet size, $H<<D$. To study the final stages of the coalescence of a bubble at a PP11-air interface, complementary experiments were conducted using a thin liquid film capillary cell (Scheludko-Exerowa cell) ${ }^{40,41}$ in which the thickness of a thin air-PP11-air film with a large surface area can be visualised using optical interference fringes.

Small bubbles and drops with low terminal velocities retain a spherical shape of diameter, $D$. At larger sizes, the bubbles and drops deform into oblate spheroid as they rise with different horizontal, $D_{\mathrm{h}}$ and vertical, $D_{\mathrm{v}}$ diameters (Figure 1c). Therefore, it is convenient to characterize the bubble or drop sizes using the equivalent diameter, $D \equiv\left(D_{\mathrm{h}}{ }^{2} D_{\mathrm{v}}\right)^{1 / 3}$. To describe the approach of a bubble or drop to the upper flat interfaces, we use the distance, $H$ between the interface and the top of the approaching bubble or drop so that apparent contact corresponds to $H=0$ (see Figure 1b).

\section{RESULTS}

We first present and discuss results from terminal rise velocity, $U_{\mathrm{T}}$ studies for small (diameter $D<230 \mu \mathrm{m}$ ) bubbles and water drops in PP11 liquid for which the rise velocity is slow and the fluid mechanics remain in the small Reynolds number, $\mathrm{Re} \equiv \rho D U_{\mathrm{T}} / \mu<0.1$ regime in which theoretical drag predictions are well-established because the bubbles/drops remain spherical. These results provide a check of the experimental procedures and parameter values and indicate whether the interfaces of these bubbles and water drops, with and without added surfactants, are 
mobile or immobile. Results from experiments using larger bubbles and water drops of diameter, $D$ up to $1300 \mu \mathrm{m}$ corresponding to Reynolds number, Re below 20, are used to quantify effects due to deformation at higher terminal velocities.

Using high speed video recording, we track the rise of bubbles and water drops that have attained their terminal velocity towards a flat PP11-air and PP11-water interface. These studies allow us to systematically examine all possible combinations of interfacial boundary conditions: mobile-mobile, mobile-immobile and immobile-immobile on the bubbles/drops and at the flat interface. Examples illustrating representative experimental runs are shown as Supplementary Videos 1 to 4 and Figure 2 shows snapshots from these videos. Video 1 (Figure 2a) shows the rise of a small spherical bubble of diameter, $D=196 \mu \mathrm{m}$ toward a PP11-air (left) or PP11-water (right) interfaces. Video 2 (Figure $2 \mathrm{~b}$ ) is similar to Video 1 with larger bubbles of diameter, $D=$ $520 \mu \mathrm{m}$, and Video 3 (Figure 2c) for the largest bubbles of diameter, $D=950 \mu \mathrm{m}$ in which case the surface deformation and thin liquid film that formed before coalescence can clearly be observed. Videos 1 to 3 track the bubble rise to the interface until it coalesces with the PP11-air interface. Video 4 (Figure 2d) shows the rise of a $D=413 \mu \mathrm{m}$ bubble towards the PP11-water interface and is shot at a lower frame rate to track the bubble coalescence with the water phase. 

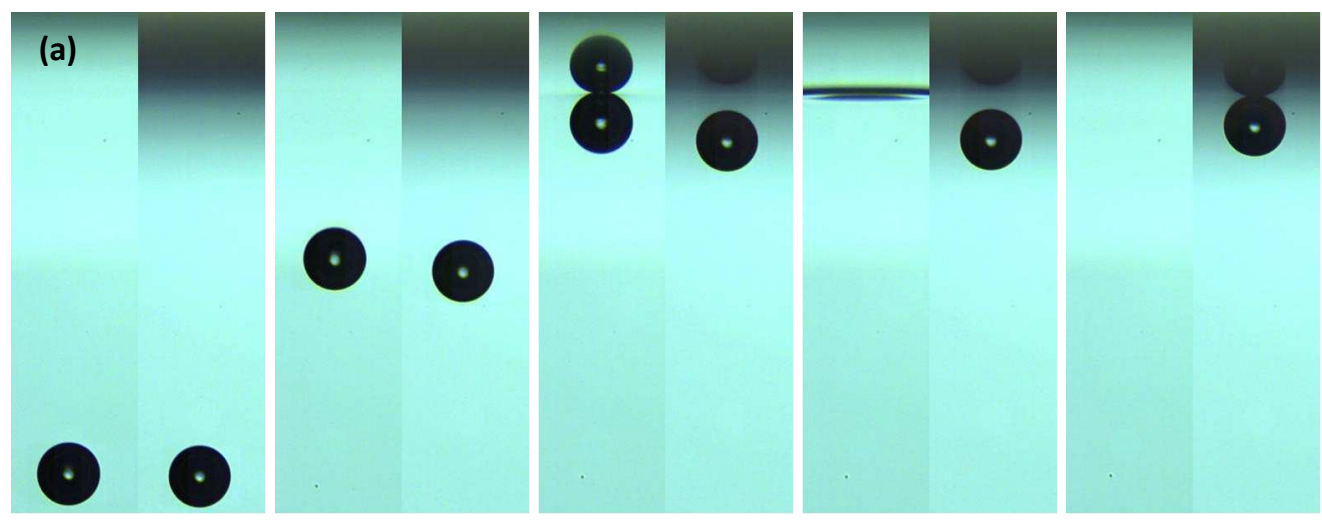

Time: $1 \mathrm{~ms}$

$155 \mathrm{~ms}$

$276 \mathrm{~ms}$

$278 \mathrm{~ms}$

$374 \mathrm{~ms}$
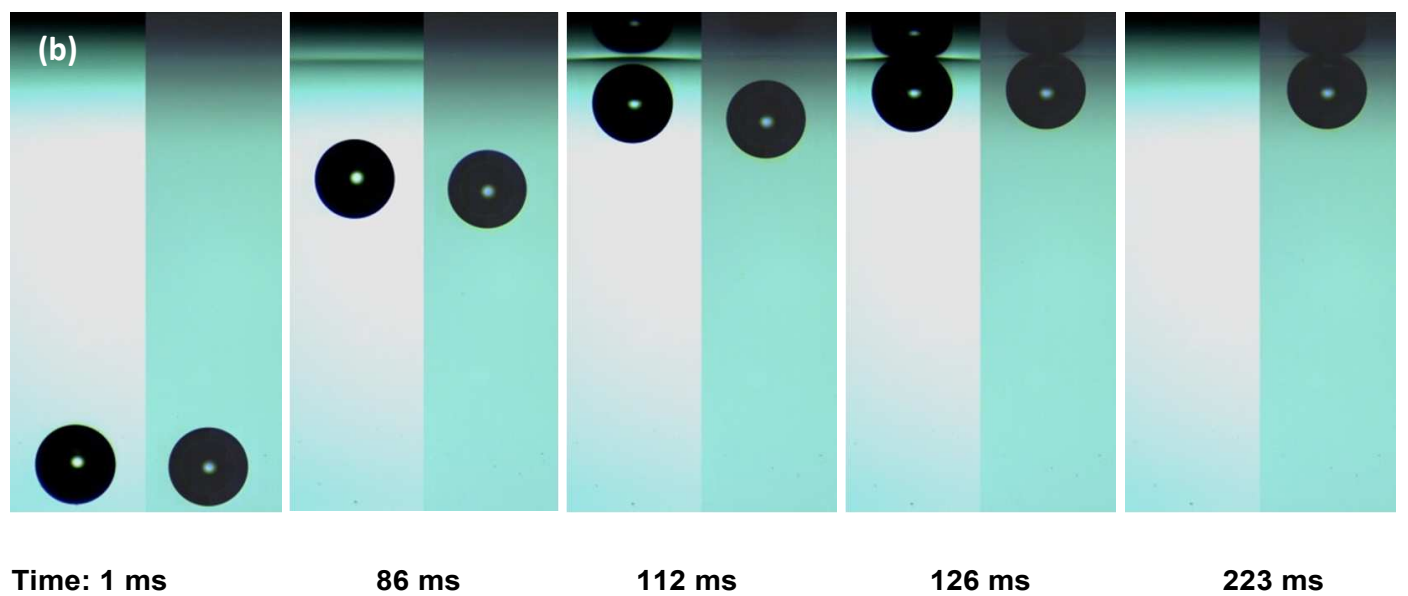

$86 \mathrm{~ms}$

$112 \mathrm{~ms}$

$126 \mathrm{~ms}$

$223 \mathrm{~ms}$

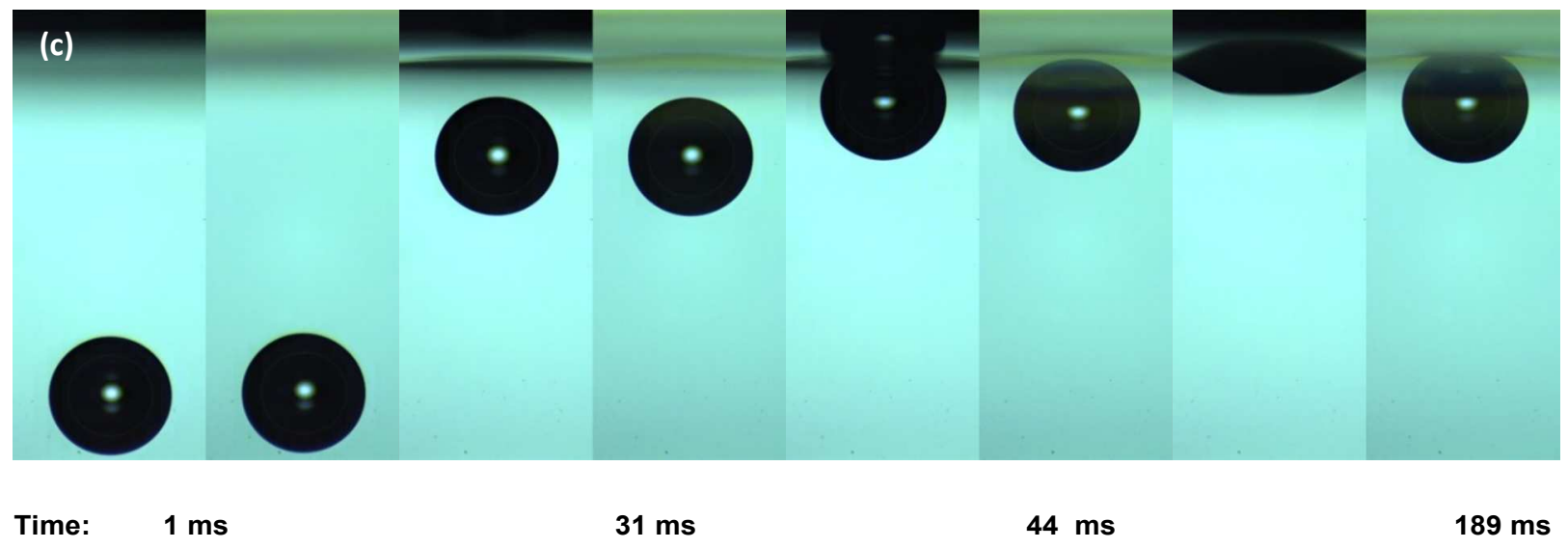




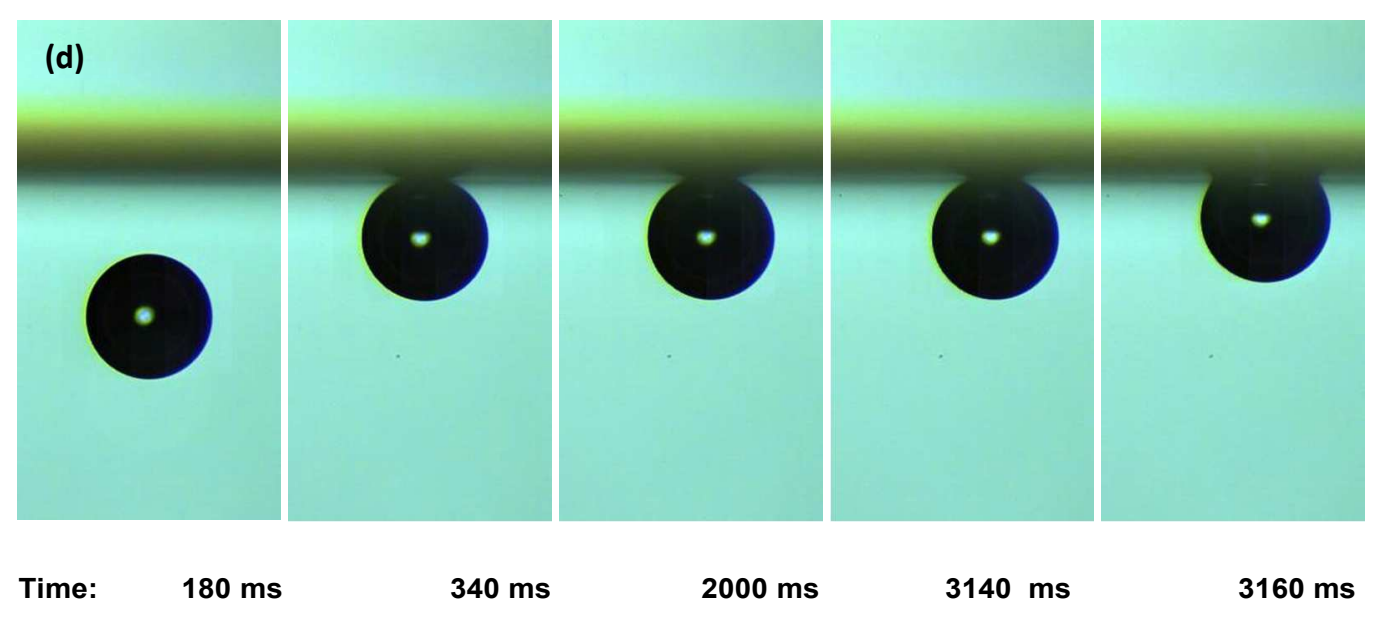

Figure 2. $(\mathrm{a}$ - c) Snap shots from Supplemental Videos 1-3 of bubbles in PP11 with a mobile interface, for different bubble sizes of (a) $D=196 \mu \mathrm{m}, \operatorname{Re}=0.08-$ Video 1 , (b) $D=520 \mu \mathrm{m}$, Re $=1.3-$ Video 2, (c) $D=950 \mu \mathrm{m}, \mathrm{Re}=6.5-$ Video 3. The bubbles rise towards a flat (left) PP11-air and (right) PP11-water interface. Note that in the last time step, the bubble has coalesced at the mobile PP11-air interface whereas at the immobile PP11-water interface, drainage of the thin PP11 film between the bubble and the PP11-water interface is still in progress. (d) Snap shots from Supplemental Video 4 of a bubble in PP11 with a mobile interface, $D=413 \mu \mathrm{m}$ rising towards the immobile PP11-water interface. The bubble arrives at the interface at $\mathrm{t}=340 \mathrm{~ms}$, and coalesces with the water phase at $\mathrm{t}=3160 \mathrm{~ms}$. Video 1-3 (a-c) were shot at a frame rate of 1000 fps whereas Video 4 (d ) was shot at 50 fps. 


\subsection{Terminal rise velocity in PP11 at $R e<0.1$}

We first consider results for small bubbles and water drops that rise at low velocities as characterized by a low Reynolds number, $\operatorname{Re}=\rho D U_{\mathrm{T}} / \mu<0.1$ and remain spherical as determined by a small Weber number, $\mathrm{We}=\rho D U_{\mathrm{T}}^{2} / \sigma<10^{-3}$, that measures the relative effects of inertia to surface tension force, where $\sigma$ is surface tension, and by a small capillary number, $\mathrm{Ca}=\mu U_{\mathrm{T}} / \sigma<$ $10^{-2}$, that measures the relative effects of viscous force to surface tension force. The three dimensionless groups are related by: $\mathrm{We}=\mathrm{Re} \mathrm{Ca}$. The terminal velocity of such small spherical bubbles will vary by up to a factor $3 / 2$ depending on whether the interface is fully mobile, with zero shear stress at the surface, or immobile, where zero relative velocity between the fluid and the surface. If the bubble or water drop interface is immobile, the terminal velocity is given by Stokes' law due to the drag force on a 'solid' sphere:

$$
U_{\mathrm{T}}=U_{\mathrm{St}} \equiv\left(\rho-\rho_{\mathrm{p}}\right) g D^{2} /(18 \mu), \quad \text { (immobile) }
$$

where $\rho$ is the PP11 density, $\rho_{\mathrm{p}}$ is the sphere density $\left(\rho_{\mathrm{p}}<<\rho\right.$ for air bubble, $\rho_{\mathrm{p}} \approx 1.0 \mathrm{~g} / \mathrm{cm}^{3}$ for water droplet) and $g$ is the gravitational acceleration. If the interface is mobile where the tangential velocity is continuous, the terminal velocity at small Re is given by the HadamardRybczynsky result, ${ }^{42-44} U_{\mathrm{HR}}$ :

$$
U_{\mathrm{T}}=U_{\mathrm{HR}} \equiv\left[\left(\mu+\mu_{\mathrm{p}}\right) /\left(2 \mu+3 \mu_{\mathrm{p}}\right)\right]\left(\rho-\rho_{\mathrm{p}}\right) g D^{2} /(6 \mu),(\text { mobile })
$$


that is larger than the Stokes' results by a factor $(3 / 2)$ in the limit of a drop with negligible viscosity, $\mu_{\mathrm{p}}<<\mu$. In the limit of a high viscosity drop, $\mu_{\mathrm{p}}>>\mu$, this gives the Stokes' law, eqn (1), for a 'solid' sphere.

In Figure 3, we present the measured terminal velocity of the bubbles of diameter, $D<$ $230 \mu \mathrm{m}$ for which $\operatorname{Re}<0.1$. Results for the terminal velocity, $U_{\mathrm{T}} v s$ bubble diameter, $D$ are given in Figure $3 \mathrm{a}$ and in Figure $3 \mathrm{~b}$ shown in scaled units as $\left(U_{\mathrm{T}} / U_{\mathrm{St}}\right)$ vs Re. The results clearly show that the terminal velocities of small bubbles closely follow eqn (2) with $\mu_{\mathrm{p}}<<\mu$, indicating that the PP11-air interface of the air bubbles is fully mobile in our experiments.

In parallel experiments, we measured the rise velocity of small water droplets in PP11 in which a high concentration $(0.1 \mathrm{wt} \%)$ of the surfactant, Zonyl FSN has been added to the aqueous phase to guarantee that the PP11-water interface is immobilized by adsorbed surfactants that lowered the interfacial tension from $47.0 \mathrm{mN} / \mathrm{m}$ to $5.6 \mathrm{mN} / \mathrm{m}$, as mentioned earlier. In contrast to the bubble data, the results shown in Figure 3 clearly indicate that the PP11-water with surfactant interface is immobile and follows eqn (1). To further examine the mobility condition at the PP11-water interface we conducted additional experiments using Millipore purified water without added surfactant. 

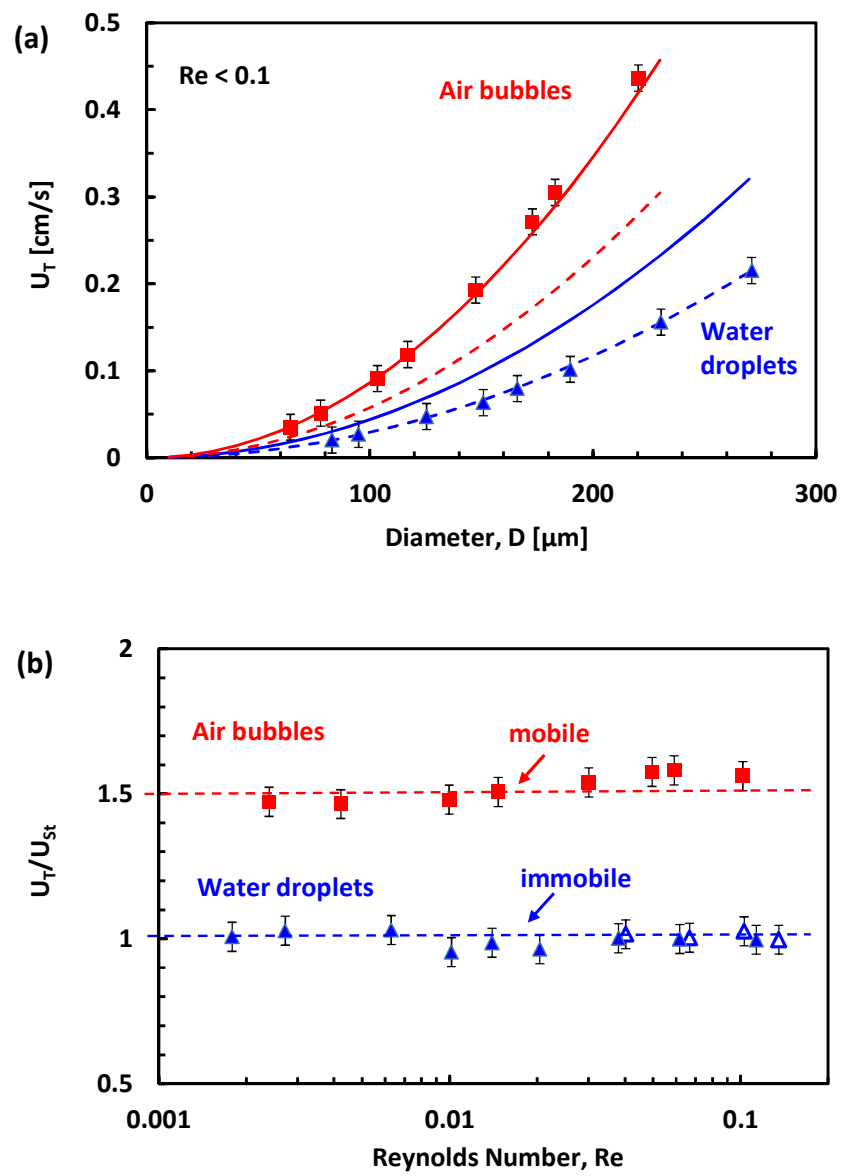

Figure 3 (a) Terminal rise velocity dependence on the bubble diameter for small $(\operatorname{Re}<0.1)$, air bubbles (solid red square) and water droplets with $0.1 \mathrm{wt} \%$ Zonyl FSN (solid blue triangles) in PP11. The solid lines are prediction for fully mobile surfaces (eqn 2) in the case of bubbles (red lines) or water droplet (blue lines) and the dash lines for the corresponding fully immobile surface case (eqn 1). (b) The same data presented as $U_{\mathrm{T}} / \mathrm{U}_{\mathrm{St}}$ as a function of Reynolds number, Re. The data points for water droplets are Millipore purified water without added surfactant (open blue triangles) or with added $0.1 \mathrm{wt} \%$ Zonyl FSN (solid triangles).

We note in Figure $3 \mathrm{~b}$ that both droplets with surfactants and droplets of pure water without added surfactants gave identical terminal velocities as a function of the droplet size. Since the 
viscosity of water is almost 19 times smaller than that of PP11, if the water-PP11 interface is mobile, the terminal velocity of small water droplets in PP11 as a function of drop size would, according to eqn (2) be within $2 \%$ of that for mobile bubbles in PP11. However, the observed results for the terminal velocity of the water drops is $2 / 3$ times that of bubbles of the same size and therefore indicate that the PP11-water interface is almost completely immobile. It is well known that even trace amounts of impurities will immobilize the oil-water interface. In our water droplets release experiments the small volume of water droplets can be easily contaminated as the water passes through the thin capillaries system used to generate the droplets. This finding is consistent with the immobile boundary condition on perfluorohexane droplets in pure water deduced from dynamic force measurements between such droplets using the atomic force microscope. $^{23}$

The laboratory measured viscosity, $\mu=19.2 \mathrm{mPa}$ of the PP11 is about $60 \%$ of the nominal value stated by the manufacturer. The two sets of data for air bubble and water droplet presented in Figure 3 together confirmed the accuracy of our measured viscosity value. For if the measured viscosity is an underestimate, it will mean that the bubbles are rising with velocities that are higher than that of a fully mobile surface bubble, eqn (2), and if it is an overestimate, it will mean that the water drops are rising with velocity lower than that predicted by the Stokes' law, eqn (1).

\subsection{Terminal rise velocity in PP11 for larger bubbles}

With high terminal velocities of larger bubbles that experience larger buoyancy forces, the hydrodynamic pressure or inertial forces will be able to deform the bubble against the surface tension force. This is characterized by the Weber number, We $=\rho D U_{\mathrm{T}}{ }^{2} / \sigma$. In Figure 4, we show 
results for the terminal velocity including larger bubbles, $D<1400 \mu \mathrm{m}$. The deformed shape of such bubbles can be approximated by an oblate ellipsoid with horizontal, $D_{\mathrm{h}}$ and vertical, $D_{\mathrm{v}}$ diameters (Figure 1c). The equivalent diameter, $D \equiv\left(D_{\mathrm{h}}{ }^{2} D_{\mathrm{v}}\right)^{1 / 3}$ provides a convenient measure of bubble size and the degree of deformation is measured by the aspect ratio: $\chi \equiv D_{\mathrm{h}} / D_{\mathrm{v}}$.
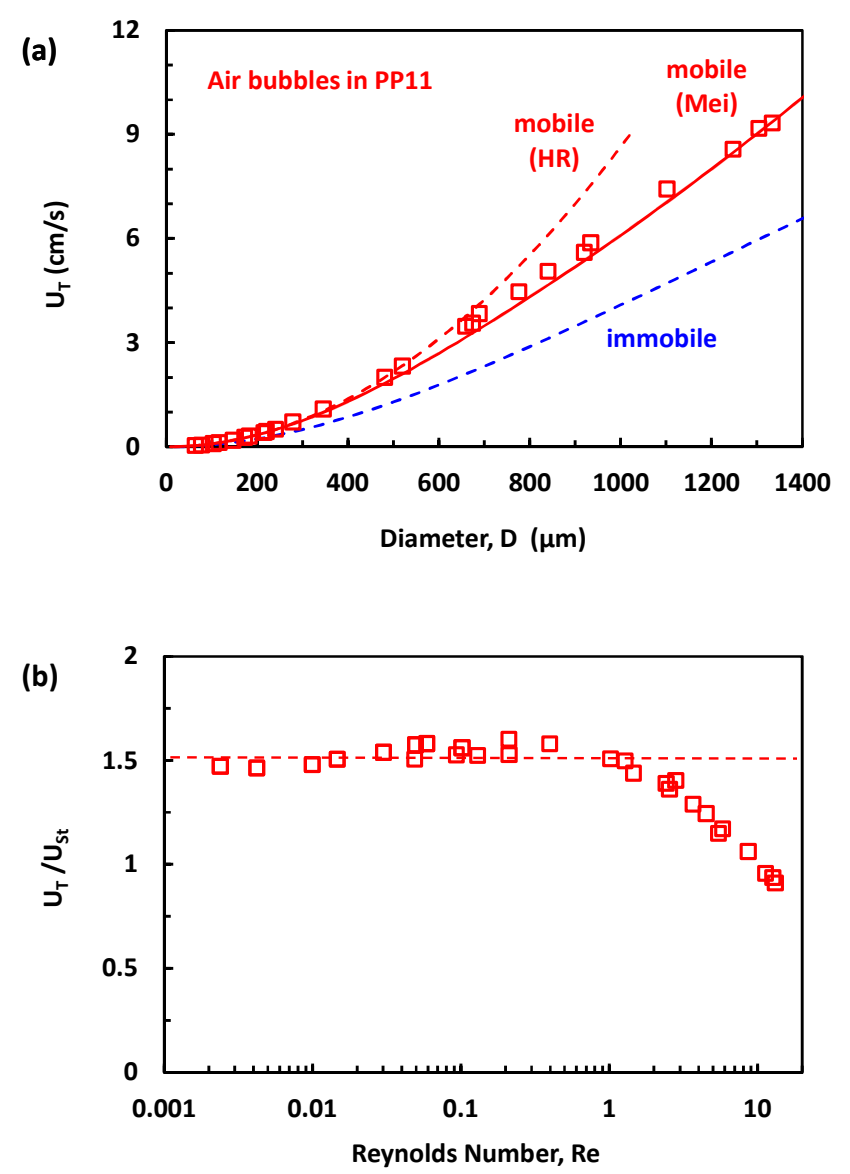

Figure 4. Terminal velocities, $U_{\mathrm{T}}$ of air bubbles free rising in PP11. (a) $U_{\mathrm{T}}$ vs. bubble diameter. The solid red line is the theoretical prediction using the correlation given by Mei et al. ${ }^{45}$ eqn 3 for mobile interface spherical bubbles, and the dashed blue line is Schiller-Naumann ${ }^{46}$ dependence for immobile interface spherical bubbles. (b) The same data presented as $U_{\mathrm{T}} / \mathrm{U}_{\mathrm{St}} \mathrm{vs}$. Re. 
We work with bubbles with $D<1400 \mu \mathrm{m}$ for which the deformation is relatively small: for $D<800 \mu \mathrm{m}, 1 / \chi>0.99$ and $\mathrm{We}<0.3$; for $D \approx 1000 \mu \mathrm{m}, 1 / \chi=0.96$; and for $D \approx 1350 \mu \mathrm{m}$, $1 / \chi=0.91$ and $\mathrm{We}=2.2$. The maximum value of the Reynolds number is up to 10 . The rising bubbles attain terminal velocity when the buoyancy force, $F_{\text {buoy }} \equiv(\pi / 6)\left(\rho-\rho_{\mathrm{p}}\right) g D^{3}$ is balanced by the drag force, $F_{\mathrm{drag}} \equiv\left(\pi \rho D^{2} U_{\mathrm{T}}^{2} / 8\right) C_{\mathrm{d}}$, to give the implicit result: $U_{\mathrm{T}}^{2}=(4 / 3)\left[\left(\rho-\rho_{\mathrm{p}}\right) / \rho\right] g D / C_{\mathrm{d}}$, since the drag coefficient, $C_{\mathrm{d}}$ varies with the Reynolds number, Re and hence $U_{\mathrm{T}}$.

In Figure 4a we compare the measured bubble rise velocities with the theoretical prediction of the drag coefficient, $C_{\mathrm{d}}$ by Mei et $a l^{45}$ for terminal velocity of fully mobile spherical bubble valid for intermediate Reynolds numbers $(1<\operatorname{Re}<50)$ :

$$
C_{\mathrm{d}}=(16 / \mathrm{Re})\left\{1+\left[8 / \operatorname{Re}+(1 / 2)\left(1+3.315 \mathrm{Re}^{-1 / 2}\right)\right]^{-1}\right\} \quad(\text { mobile })
$$

, and for fully immobile bubbles surface can be estimated using the Schiller-Naumann) ${ }^{46}$ empirical relation valid for $0.2<\operatorname{Re}<1000$ :

$$
C_{\mathrm{d}}=(24 / \mathrm{Re})\left(1+0.15 \mathrm{Re}^{0.687}\right) \quad \text { (immobile) }
$$

It is clear that the measured terminal velocities of rising bubbles in PP11 are consistent with a mobile interface in agreement with the earlier low velocity results with smaller bubbles. The effects of the small bubble shape deformation are not significant in this size range. The experimental results are not in the very large Reynolds number regime as in previous studies ${ }^{21}$ of bubbles in water that are in agreement with the Moore model ${ }^{47}$ that is valid for $\operatorname{Re}>100$. 
The same results presented in Figure $4 \mathrm{~b}$ indicate that the bubble terminal velocity can be approximated by the simple Hadamard-Rybczynsky formula, eqn (2), up to $\operatorname{Re}<1$, that correspond to the bubble diameter, $D<500 \mu \mathrm{m}$ in PP11.

\subsection{Bubbles and water drops colliding with PP11-air and PP11-water interfaces at $\operatorname{Re}<\mathbf{0 . 1}$}

We now examine the collision dynamics of bubbles and water droplets in PP11, as they collide with a PP11-air or PP11-water interface after having reached their terminal rise velocity in the regime $\operatorname{Re}<0.1$. The results will quantify the different effects of 4 combinations of boundary conditions:

i) mobile bubble against mobile PP11-air

ii) mobile bubble against immobile PP11-water

iii) immobile water drop against mobile PP11-air

iv) immobile water drop against immobile PP11-water

In this low Reynolds number $(\operatorname{Re}<0.1)$ and small size regime, the bubbles $(D<200 \mu \mathrm{m})$ and drops $(D<300 \mu \mathrm{m})$ remain spherical and the flat PP11-air and PP11-water interfaces deformation is also negligible until the bubble or droplet reaches the surface (Supplemental Video 1 and Figure 2a). In the same regime, a general theoretical model developed by Bart ${ }^{45}$ is available for the hydrodynamic force between the interface and the bubble or drop that will allow us to model the deceleration as the bubbles or drops approach the flat interface under a constant buoyancy force. In the Bart model, valid in the limit of Re approaching 0, the bubble or drop is assumed to be a sphere with arbitrary internal viscosity that travels towards a planar fluid-fluid 
interface that has a general viscosity ratio between the two phases. The geometry is shown in Fig. 1b.

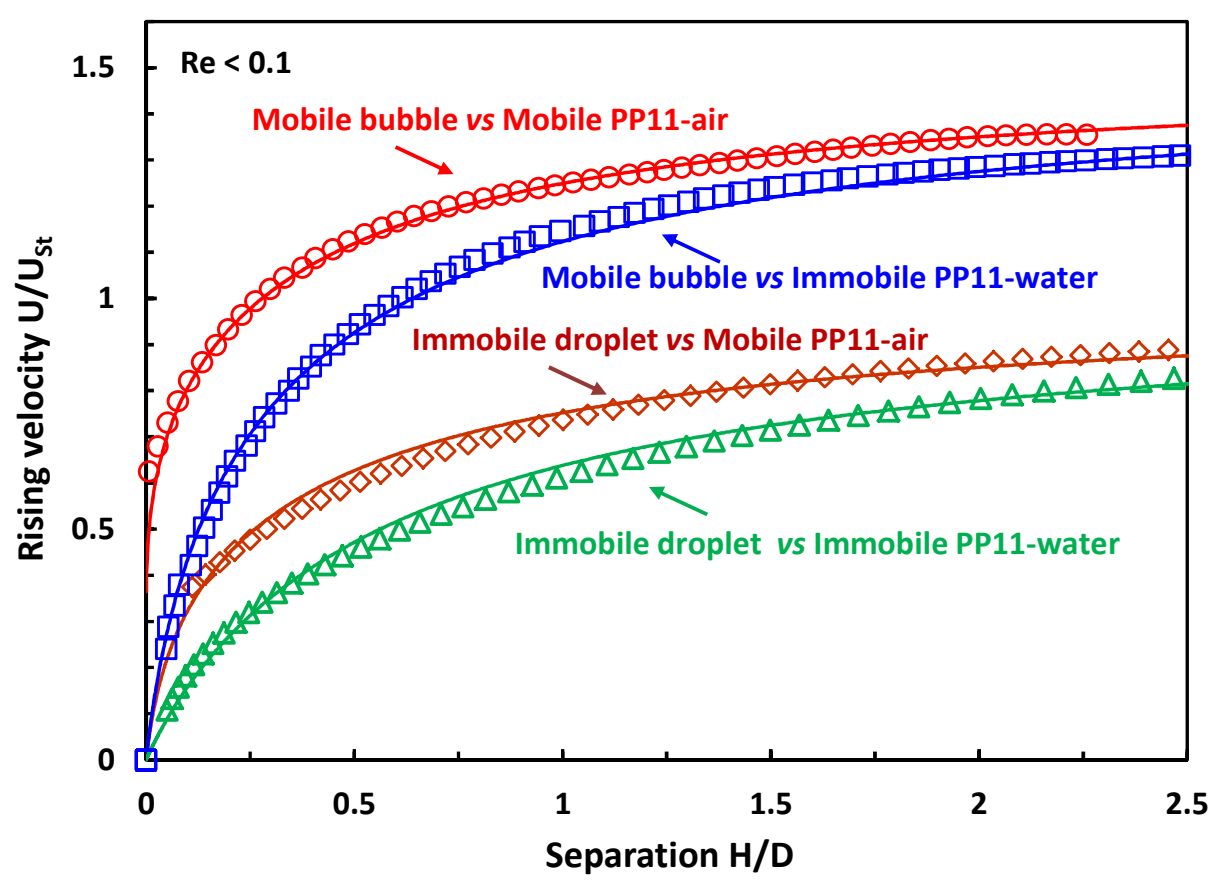

Figure 5. Comparison of the rising velocity, $U / U_{\mathrm{St}} v s$ separation, $H / D$ experimental data (symbols) with the Bart theory (solid lines) for the four cases of: air bubble $D=196 \mu \mathrm{m}$, rising toward a free PP11 surface (red circles, red line); air bubble $D=198 \mu \mathrm{m}$ rising toward PP11water interface (blue squares, blue line); water droplet $D=254 \mu \mathrm{m}$ rising toward free PP11 interface (brown diamonds, brown line); water droplet $D=300 \mu \mathrm{m}$ rising toward PP11 - water interface (green triangle, green line). In all cases, $\operatorname{Re}<0.1$.

Figure 5 provides a comparison between our experimental data obtained from analyzing high speed video recordings and predictions based on the Bart theory ${ }^{48}$ for the deceleration kinetics of small air bubbles or water drops with $\operatorname{Re}<0.1$, approaching the liquid interface. The velocity, 
scaled by the Stokes' velocity, $U_{\mathrm{St}}$ given by eqn (1), is shown as a function of the separation, $H$ between the flat surface and the top of the bubble or drop scaled by the diameter, $D$ (Figure $1 \mathrm{~b}$ ).

Bart's theory ${ }^{48}$ gives an expression for the hydrodynamic force, $F_{\text {hy }}$ between a sphere of diameter, $D$ and a flat interface as a function of the separation, $H$ and the instantaneous normal velocity of the sphere $d H / d t$ that can be expressed as:

$$
F_{\mathrm{hy}}=-3 \pi \mu D \lambda(H)(d H / d t)
$$

, so that the force is repulsive, $F_{\text {hy }}>0$, if the sphere approaches the flat interface, $d H / d t<0$. Bart gave results for the function $\lambda(H)$ as infinite series for all four combinations of mobile and immobile boundary conditions on the sphere and on the flat interface. Far from the surface, $H \rightarrow$ $\infty, \lambda(H) \rightarrow 1$ if the sphere has an immobile boundary and $\lambda(H) \rightarrow 2 / 3$ if the sphere has a mobile boundary.

The equation of motion of the bubble is obtained by equating the hydrodynamic force to the buoyancy force:

$$
3 \pi \mu D \lambda(H)(d H / d t)=-(\pi / 6)\left(\rho-\rho_{\mathrm{p}}\right) g D^{3}
$$

, and by starting with a sphere at a large distance from the interface with the known terminal velocity, $U_{\mathrm{T}}$, we have the bubble speed, $U=|d H / d t|$ as a function of the separation, $H$. Such theoretical results are shown as solid curves in Figure 5. Thus for $\operatorname{Re}<0.1$, there is excellent agreement between the experimental results and the theoretical predictions, confirming that in our experiments the free PP11-air interface is fully mobile surface whereas the PP11-water interface is fully immobile. Note that in the present small Re limit, the effect of the presence of the flat interface is quite long-ranged as the bubbles and drops begin to slow down at around 5 diameters away and start to decelerate. 
We note that Bart made a similar comparison between theory and experiment using low viscosity ethylene glycol drops in a very high viscosity fluid (UCON LB 3000X) in order to remain in the low Re regime. ${ }^{48}$ However, technical limitation at the time resulted in only modest agreement between theory and experiments in some cases. To the best of our knowledge the comparisons in Figure 5 are the most precise confirmation of Bart theoretical predictions for the four combinations of boundary conditions considered here. More importantly they demonstrate that a bubble with a mobile surface rising toward a clean mobile liquid interface will decelerate significantly slower compared to the same bubble rising toward an immobile interface. The difference in the deceleration of a mobile bubble approaching a mobile PP11-air interface and an immobile PP11-water interface is illustrated in the Supplemental Video 1 and snapshots from this video which are shown in Figure 2a. The video also demonstrates that during the approach of the mobile bubble there is negligible deformation of the mobile flat PP11-air interface or the immobile flat PP11-water interface. These results are consistent with the finding that the bubble surface in PP11 is mobile whereas the surface of a water drop (with or without Zonyl FSN surfactant) in PP11 is immobile.

The results for the terminal velocity of bubbles rising in PP11 and bubbles decelerating toward a PP11-air interface (Figures 3 - 5) were very reproducible and insensitive to the history of the PP11-air interface. For example if a bubble formed at the tip of the micro-capillary was first held there for hours before being released to rise, the terminal velocity was still identical to that of bubbles that were immediately released upon formation. Similarly, the use of a freshly formed PP11-air surface (by pouring or by sucking to clean the top surface) gave the same results as experiments with surface exposed to the laboratory air for days. This is in sharp 
contrast to experiments conducted with bubbles in water or water-air free interfaces known to exhibit rapid aging and immobilizing when exposed to air for even a matter of minutes. ${ }^{8}$

\subsection{Larger bubbles colliding with PP11-air and PP11-water interfaces}

Next we consider results of experiments using larger bubbles $250 \mu \mathrm{m}<D<1400 \mu \mathrm{m}$, in which we compare the collision dynamics of rising bubbles at the terminal velocity towards either a mobile PP11-air surface or an immobile PP11-water interface. Videos corresponding to the case of $D=520 \mu \mathrm{m}$ bubbles (Supplemental Video 2) and for $D=950 \mu \mathrm{m}$ bubbles (Supplemental Video 3) illustrate a similar difference in the deceleration when approaching a mobile PP11-air interface compared to an immobile PP11-water interface as for smaller bubbles.

For experiments with increasingly higher Reynolds numbers, the results are expected to gradually deviate from the Bart theory, as seen in Figure 6a for $U / U_{\mathrm{St}} v s H / D$ at $D=520 \mu \mathrm{m}, \operatorname{Re}$ $=1.3$. For larger bubble sizes the flat interface is observed to deform significantly once the bubbles get close to the surface $(H / D<1.0$, Supplemental Videos 2 and 3). Nevertheless, in each case bubbles that rise toward the mobile PP11-air interface slow down less rapidly when compared to those approaching an immobile PP11-water, as shown in Figure 6b, where the data for the largest bubbles, $D=647 \mu \mathrm{m}$ and $D=1000 \mu \mathrm{m}$ are now presented as the variation of the bubble center of the mass velocity with time.

Results for the terminal rise velocities presented in the previous sub-sections demonstrate that bubbles in the small $(D<240 \mu \mathrm{m}, \mathrm{Re}<0.1)$ and in the intermediate Reynolds numbers range $(D<1400 \mu \mathrm{m}, \operatorname{Re}<20)$ have fully mobile surfaces. The deceleration kinetics of small bubbles $(\operatorname{Re}<0.1$, We $<0.1)$ rising toward the flat surface indicate that the flat PP11-air interface is also fully mobile whereas the flat PP11-water interface is immobile. The 
experimental results for the deceleration of small $(\operatorname{Re}<0.1)$ and larger $(\operatorname{Re}>0.1)$ bubbles follow the same trends in relation to the effects of the mobility of the flat P11-air and PP11-water interfaces.
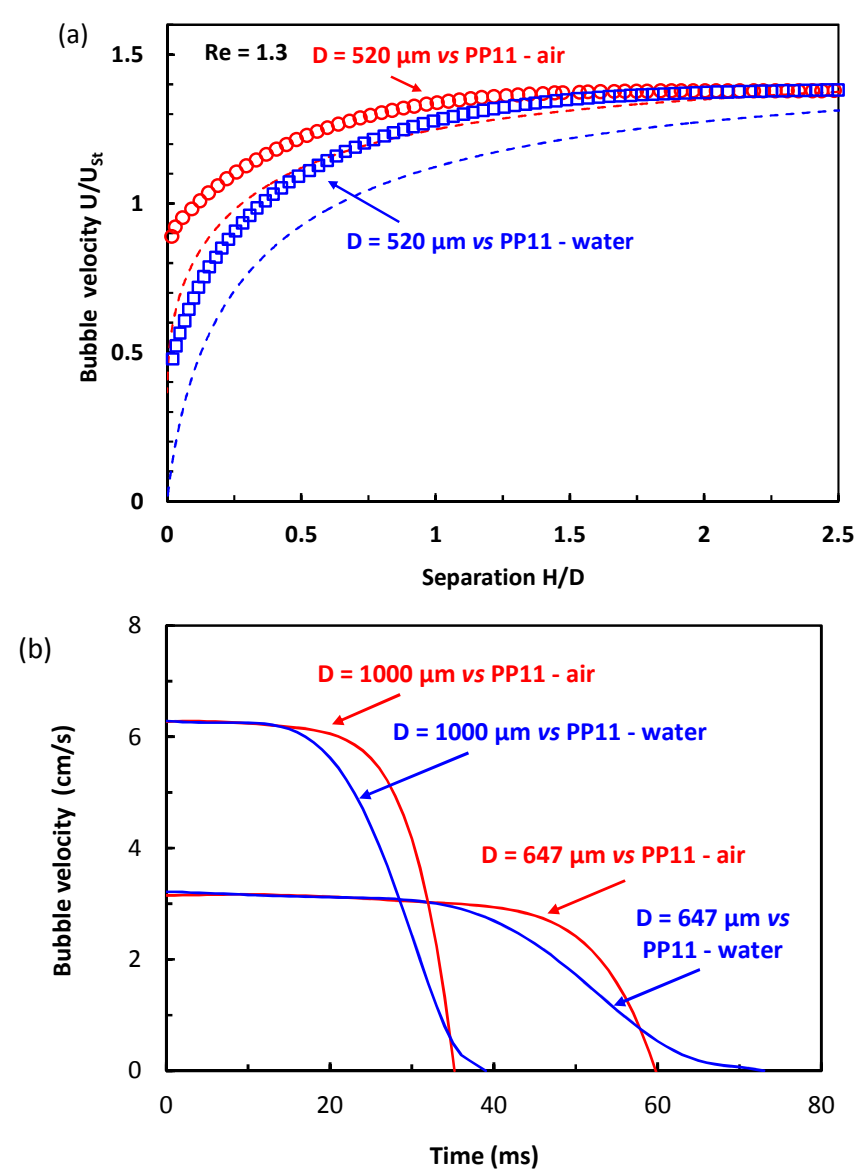

Figure 6. (a) Velocity, $U / U_{\mathrm{St}} v s$ separation, $H / D$ experimental data for the cases of air bubble $D$ $=520 \mu \mathrm{m}$, rising toward PP11 - air surface (red circles) or toward PP11-water interface (blue squares). In this case away from the surface, the bubble $\mathrm{Re}=1.3$, and the results deviate from the Bart theory predictions for $\mathrm{Re}<<1.0$ shown as dotted lines (red for PP11-air and blue for PP11water). (b) Experimental bubble center of mass velocity $v$ s time data for the case of air bubble of $D=647 \mu \mathrm{m}$, or $D=1000 \mu \mathrm{m}$ rising toward PP11-air surface (red lines) or PP11-water interface 
(blue lines). For the $D=647 \mu \mathrm{m}$ bubbles the zero time is set at $H / R=5.0$ and for the $D=1000$ $\mu \mathrm{m}$ bubbles at $H / R=3.5$.

\subsection{Bubble induction times before coalescence}

Supplemental Videos 1-4, provide graphic examples for the coalescence of bubbles with the free PP11-air surface or PP11-water interface. One can see that there is an observable delay between the bubbles arrival at the interface and the actual coalescence event. These delays are much shorter for the case of mobile PP11-air compared to the immobile PP11-water interface. This time delay is referred to as the induction time or the film drainage time, reflecting the time taken for the intervening thin PP11 film between the bubble and the flat surface to drain before coalescence occurs. Although there might be some uncertainty as to the exact moment the bubble reached the flat interface, providing that the induction time is long, this uncertainty is not important.

The term induction time is commonly used in the context of describing particle capture by bubbles in, for example, mineral flotation applications. In general, apart from the time taken for the thin liquid film to drain between the particle and the bubble, other dynamic effects such as the rearrangement of adsorbed polymeric species can also contribute to the induction time. In the present paper, thin film drainage is the dominant mechanism that contributes to the induction time.

The variation of the experimental induction time with bubble size for coalescence with the mobile PP11-air interface or with the immobile water-PP11 interface is summarized in Figure 7. In all experiments the bubbles were released far enough from the surface to reach terminal velocity before the collision. 
At the mobile PP11-air interface, bubbles of diameter smaller than $240 \mu \mathrm{m}$ coalesce almost immediately upon arriving at the surface (Supplemental Video 1). For larger bubbles, liquid film drainage time could be measured readily (see Supplemental Videos 2 and 3). The clear trend is that the induction time $\tau$, gradually increase with the bubble size: $\tau=0.01 \mathrm{~s}$ for $D=$ $330 \mu \mathrm{m} ; \tau=0.1 \mathrm{~s}$ for $D=800 \mu \mathrm{m} ; \tau=0.3 \mathrm{~s}$ for $D=1300 \mu \mathrm{m}$. Empirically, $\tau$ appears to increase with $D^{2}$ over this bubble size range (Figure $7 \mathrm{~b}$ ). The induction time results were very reproducible with minimal scatter in the measured values across numerous experiments. As in the case of bubble rise velocities the results were unaffected by the surface aging of the bubble or the PP11-air flat interface. In some cases we first added water with a higher concentration of F surfactant Zonyl FSN on top of the PP11 and then the water phase was removed by sucking it from the top to create a fresh PP11-air interface for the coalescence experiments. In such cases, the measured drainage times were identical to that using a freshly created PP11-air interface. This finding confirms the exceptional resistance of PP11 to surface active impurities.

In contrast, for bubbles rising towards an immobile PP11-water interface, the induction or coalescence times were more than two orders of magnitudes longer than for the bubble coalescence at the mobile PP11-air interface as shown in Figure 7. To minimize the PP11-water interfacial charge, an aqueous electrolyte $(0.5 \mathrm{M} \mathrm{NaCl}$ electrolyte at $\mathrm{pH} 3.0)$ was always used as the upper phase. Over the range of bubble radii examined, the coalescence times had a scatter of over an order of magnitude. This large spread of induction times is characteristic for films in which one of the phases is water ${ }^{49,50}$ and is usually attributed to the susceptibility of the air-water or oil-water interface to surface active contaminants. A contributing factor in our experiment can be the fact that the bubble is found to slowly migrate horizontally during the drainage phase as can be noticed in Supplementary Video 4 and Figure 2d. The detailed reason for the poor 
reproducibility of the drainage times in the case of coalescence at the PP11-water interface might be more complex and should be clarified in further investigations. In the context of the present study we use these experiments only as reference to indicate a significant difference in the induction time for the coalescence of mobile bubbles at an immobile interface (PP11-water) compared to that at a mobile interface (PP11-air).
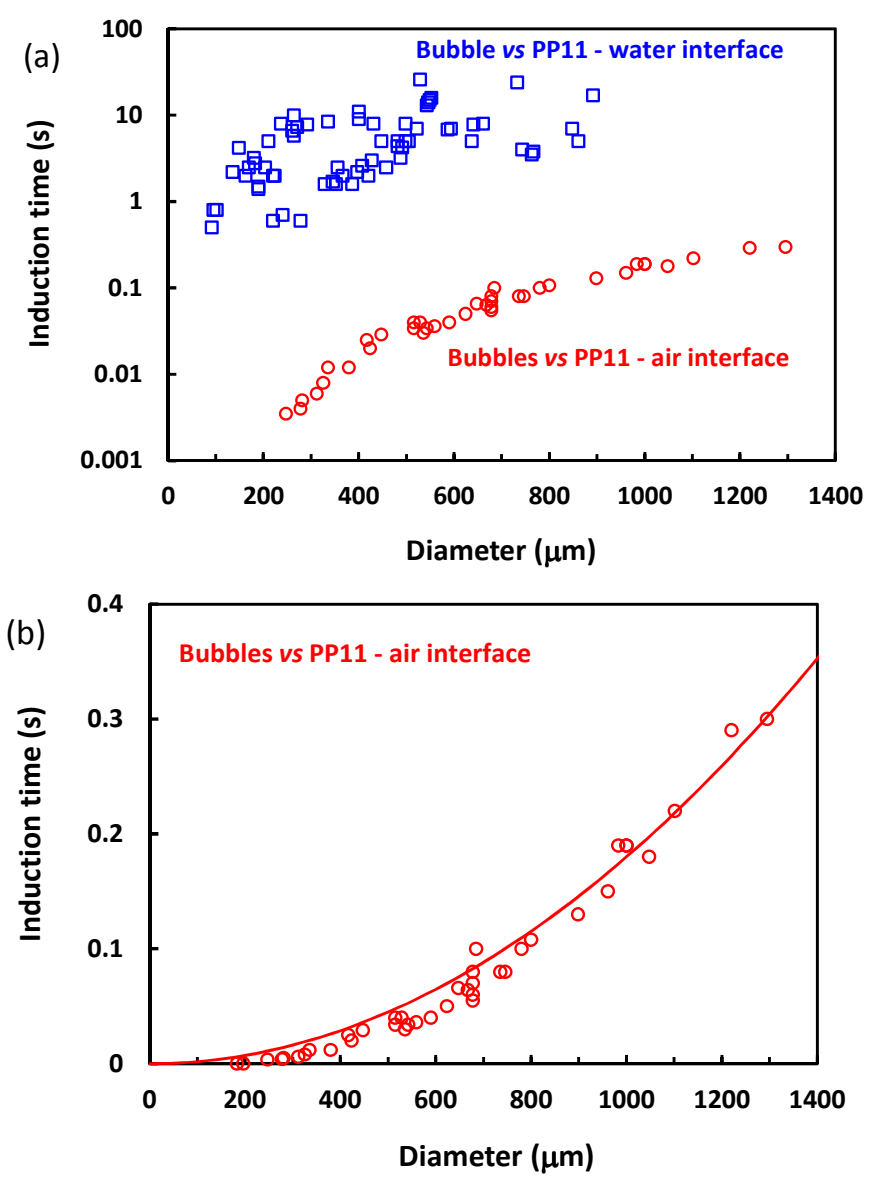

Figure 7. (a) Induction time dependence on the bubble diameter for the case of mobile bubbles rising toward a free PP11-air surface (red circles), or PP11-water with $0.5 \mathrm{M} \mathrm{NaCl}$ at pH 3.0 (blue square). Data for each case are collected from multiple experiments (conducted at different days). (b) Induction time dependence on the bubble diameter at the free PP11-air surface plotted vs. linear time scale. The solid line is a fit to a $\tau \sim D^{2}$ dependence. 
We can speculate that the key reason for the large difference in induction time at the mobile PP11-air interface and at the immobile PP11-water interface is the difference in the interfacial mobility. A secondary reason could be the difference in the van der Waals force between the two systems: attractive for the case of air-PP11-air film, and close to zero for air-PP11-water films because water and PP11 have the same refractive index. However, the van der Waals forces are relatively short-ranged and in the following estimate, the magnitude of the van der Waals attraction is insufficient to account for the much shorter induction times of the air-PP11-air films. We also notice that the PP11-air surface tension $(\sigma=21.5 \mathrm{mN} / \mathrm{m})$ is less than half of the PP11water interfacial tension $(\sigma=47.0 \mathrm{mN} / \mathrm{m})$, but the effect of a more rigid surface is expected to only decrease the induction time.

\subsection{Estimation of the air-PP11-air film rupture thickness}

With the present experimental arrangement of a bubble approaching a PP11-air interface, it is not possible to measure directly the characteristic thickness at which the thin PP11 film ruptures and triggers the coalescence process. This thickness is of course related to the induction or film drainage time. However, using a thin liquid film cell (Scheludko-Exerowa cell, $3.6 \mathrm{~mm}$ diameter) we can observe directly the formation and rupture of PP11 thin films surrounded by air on both sides. The measured film lifetime of 0.1 to 0.3 seconds was in the same range as the induction times encountered in the bubble collision with PP11-air surface experiments. Although this short lifetime did not permit detailed tracking of the film thinning kinetics, the formation of a uniform black film was always clearly observed prior to film rupture, as shown in Figure 8. The appearance of the black film indicates that the characteristic film rupture thickness is less than one quarter of the wavelength visible light, or less than $100 \mathrm{~nm}$. As discussed below, 
attractive van der Waals forces are expected to be the main cause of rupture for such thin airPP11-air films.

Since the refractive indices of PP11 and water are practically identical in the visible region of the spectrum, an interferometric estimate of the film thickness at rupture is problematic for the air-PP11-water film. However, this close index match means that the magnitude of the van der Waals interaction will be much smaller for the air-PP11-water system than the air-PP11air system. Hence, the rupture thickness of air-PP11-water films should be well below the experimental estimate of the rupture thickness of air-PP11-air films discussed above.

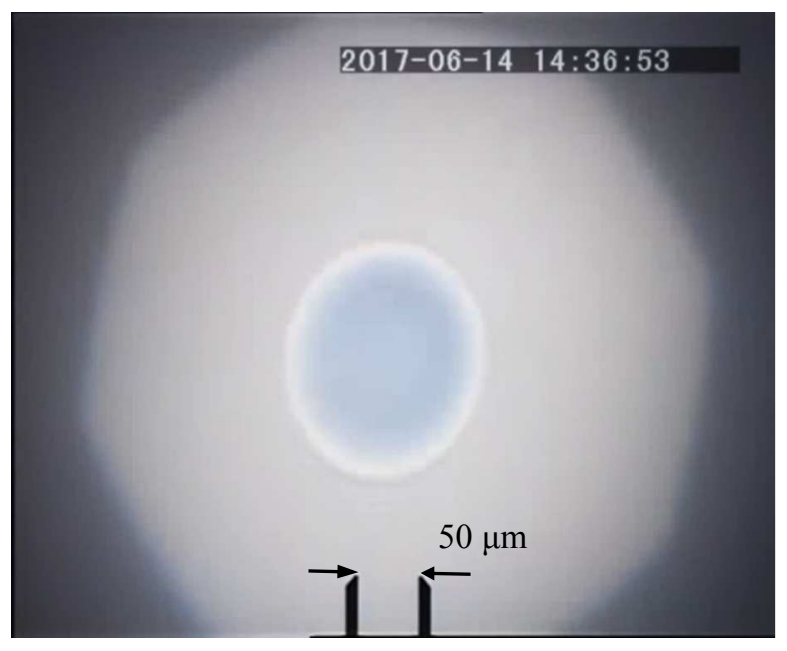

Figure 8. Video snapshot of thin liquid film of PP11 observed in a capillary cell, taken just prior to film rupture. The appearance of a uniform dark film of diameter $\sim 100 \mu \mathrm{m}$ indicates that the film rupture thickness was $<100 \mathrm{~nm}$, one quarter of wavelength of visible light in PP11. 
With these preliminary remarks we can explore the different coalescence times with simple theoretical modelling in order to gain some physical insight into the role of the hydrodynamic on the film drainage process and of the surface forces in triggering coalescence.

\section{DISCUSSION}

\subsection{Bubble coalescence at the air-PP11-air interface}

The most significant feature of the experimental results in Figure 7 for the induction times of a bubble in PP11 coalescing at the PP11-air interface and at the PP11-water interface is the 2 orders of magnitude difference between the two cases. For bubble coalescence at the PP11-air interface, the variation of the induction time with bubble diameter, $D$ is very reproducible and appears to increase with $D^{2}$ for diameters in the range $200 \mu \mathrm{m}$ to $1300 \mu \mathrm{m}$. For smaller bubbles, the induction times were too short to be measurable. In contrast, the induction times for bubble coalescence at the PP11-water interface are 2 orders of magnitude larger and exhibit considerable scatter, as much as over a factor of 10 , and the data only suggest weak size dependence.

The terminal rise velocities of bubbles and water droplets presented above indicate that the PP11-air interface is fully mobile whereas the PP11-water interface is immobile. To demonstrate that this difference in hydrodynamic boundary condition is responsible for the observed large difference in both cases we carried out the following calculation.

Bubble coalescence at the PP11-air interface involved the thinning and eventual rupture of the air-PP11-air film. In this simple system, the van der Waals (vdW) interaction is responsible for triggering film rupture. The attractive vdW disjoining pressure as a function of 
film thickness, $h$ can be represented as: $\Pi(h)=-A(h) /\left(6 \pi h^{3}\right)$. Effects of electromagnetic retardation means that the Hamaker function, $A(h)$ decreases with separation in a complicated manner. For our present discussion, we only require an estimate of the upper bound of the effects of the vdW interaction so we use a constant upper bound value of: $A=5 \times 10^{-20} \mathrm{~J}^{51}$

Making the assumption that one of the interfaces of the air-PP11-air film is immobile and the other is mobile, we calculated the time evolution of the film thinning process using the StokesReynolds-Young-Laplace ${ }^{1,21}$ model of film drainage using the experimental bubble velocity as input condition for four different bubble sizes. The results shown in Figure 9 indicate that if one interface of the air-PP11-air is assumed to be immobile, the film rupture or induction times, using an upper bound estimate of the magnitude of the destabilizing vdW attraction to minimize the calculated induction time, will be between $20 \mathrm{~s}$ to $1000 \mathrm{~s}$. Experimentally the observed induction time for air-PP11-air films are all less than 1 second. Therefore these modelling results clearly demonstrate the key role of interfacial mobility or hydrodynamic boundary condition on the induction time.

The direct modelling of the induction time due to drainage of a film with 2 mobile interfaces requires a substantially different and more complex theoretical model ${ }^{52}$ than that of the StokesReynolds-Young-Laplace approach. ${ }^{1,21}$ It is clear from the video clips of the coalescence events (Supplemental Video 2 and Video 3), deformation of the PP11-air interface is significant for the bubble sizes considered here due to the large buoyancy force that drive the bubbles towards the interface. This also has the consequence that the Reynolds number corresponding to the bubble dynamics is in an intermediate range, $\operatorname{Re} \sim 1-10$, so that any quantitative model will need to include these features and be valid in the relevant parameter range. We therefore defer consideration of such possible models to a future publication. 

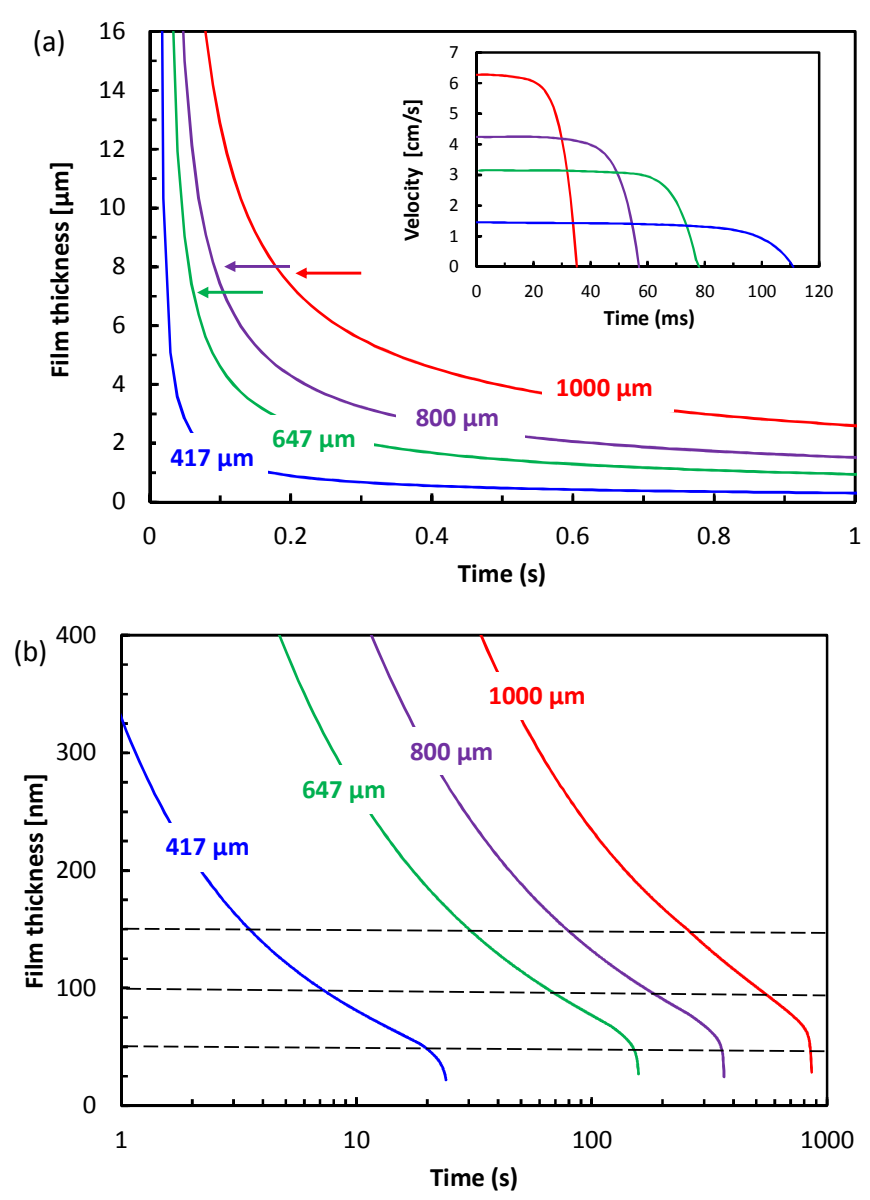

Figure 9. Calculations of the drainage of the air -PP11-air thin liquid film with time assuming one interface is immobile using the P11 surface tension, $\sigma=21.5 \mathrm{mN} / \mathrm{m}$, Hamaker constant $A=5$ $\times 10^{-20} \mathrm{~J}$. Shown are (a) short time and (b) long time results for bubble sizes: $D=417 \mu \mathrm{m}$ (blue line); $647 \mu \mathrm{m}$ (green line); $800 \mu \mathrm{m}$ (violet line); $1000 \mu \mathrm{m}$ (red line). The inset in (a) shows the experimental velocity profiles used to determine the initial boundary condition for the simulation. Arrows in (a) mark the experimentally observed induction times and corresponding simulated film thicknesses. The horizontal dashed lines in (b) indicate film thickness at $50 \mathrm{~nm}$, $100 \mathrm{~nm}$ and $150 \mathrm{~nm}$. 


\subsection{Bubble coalescence at the air-PP11-water interface}

At a qualitative level at least, the long induction times of bubble coalescence at the airPP11-water interface shown in Figure 7 can be understood in terms of the significantly longer film drainage time due to the immobile PP11-water interfaces. Electrical double layer effects have been suppressed using high salt and low $\mathrm{pH}$ in the aqueous phase. Also because of the near refractive index match between water and PP11, the magnitude of the van der Waals interaction, that is expected to trigger coalescence, will be around 2-3 orders of magnitude smaller than that for the air-PP11-air system. ${ }^{23}$ so the PP11 film thickness at which the bubble coalesces at the PP11-water interface must be much thinner than coalescence at the PP11-air interface.

We quantify this difference by using the Stokes-Reynolds-Young-Laplace ${ }^{1,21}$ model to study the thinning dynamics of the PP11 film between a PP11-air and a PP11-water interface. We take the fully mobile PP11-air interface to have surface tension, $\sigma=21.5 \mathrm{mN} / \mathrm{m}$ and the immobile PP11-water interface to have surface tension, $\sigma=47.0 \mathrm{mN} / \mathrm{m}$. For simplicity we set the van der Waals interaction to be zero in modelling the time evaluation of the thinning PP11 film (Figure 10a). We then determined the film thicknesses at film rupture and coalescence that correspond to the experimentally observed coalescence times (Figure 10b).

From the results in Figure 10, we note that the experimentally observed induction times for bubble coalescence at the PP11-water interface fall between $1 \mathrm{~s}$ to $10 \mathrm{~s}$, with considerable scatter in the bubble diameter range of $100 \mu \mathrm{m}$ to $800 \mu \mathrm{m}$. In very broad terms, such times would correspond to film rupture occurring at thickness in the range $100 \mathrm{~nm} \pm 50 \mathrm{~nm}$. Given the forgone discussion, this is an unexpected result. As the air-PP11-water van der Waals interaction is expected to be much smaller than that for air-PP11-air, it is difficult to see how such a small interaction can trigger film rupture at a film thickness of around $100 \mathrm{~nm}$. 
The other unexpected observation is the large scatter in induction times for the air-PP11water system compared to the air-PP11-air case. The PP11-water interface is expected to be molecularly smooth with uniform properties along the interface. The high gas solubility in perfluorocarbon liquids and the magnitudes of various interfacial tensions would preclude the formation of gas bubbles at the interface as air bubbles would be unable to satisfy the Neumann triangle condition. Clearly the PP11 liquid is hydrophobic, but any long range hydrophobic interaction is expected to only operate on the water side of the interface but not in the PP11 phase. Until there is evidence to the contrary, the role of interfacial contaminants that originate in the aqueous phase is implicated in triggering coalescence at the PP11-water interface with a large scatter of induction times.

Finally, notwithstanding the possible presence of interfacial contaminants, our experiments have demonstrated that the hydrophobic PP11-water interface obeys the immobile hydrodynamic boundary condition, consistent with findings using entirely different experimental techniques. $^{23}$ Therefore, the invoked conjecture that hydrophobic surface automatically result in hydrodynamic slip ${ }^{35}$ needs to be revisited and care needs to be exercise to account for other causes that give rise to the observation of apparent slip such as surface roughness, surface contaminants and trapped interfacial bubbles. 

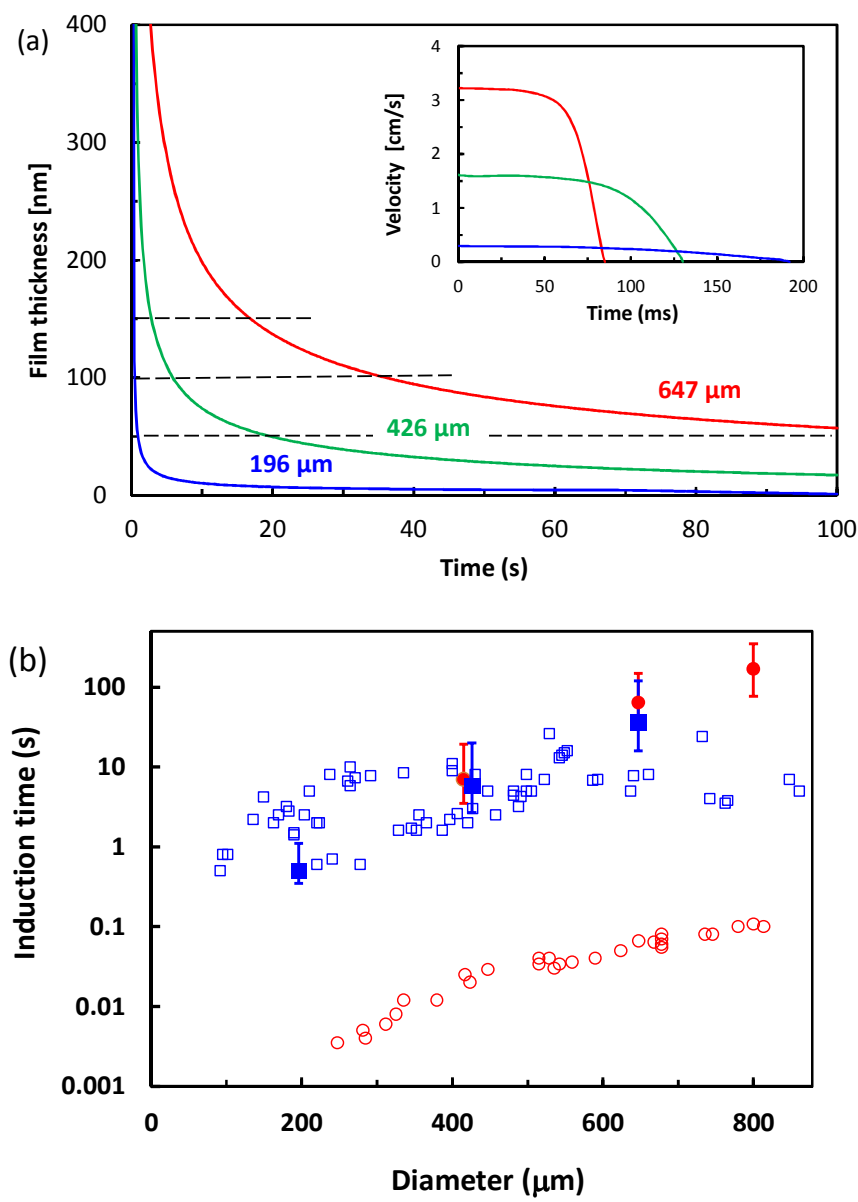

Figure 10. (a) Simulation of the air - PP11 - water thin liquid film thicknesses evolution with time assuming deformable mobile bubble surface and immobile PP11 - water interface. Air P11 surface tension is $\sigma=21.5 \mathrm{mN} / \mathrm{m}$ and PP11 - water interfacial tension is $\sigma=47.0 \mathrm{mN} / \mathrm{m}$. The insert shows the experimental velocity vs. time data used to determine the initial boundary condition. (b) Comparison of the experimental induction times for air bubble vs free PP11 interface (empty circles) or air bubbles vs. PP11-water interface (empty squares) interface with the simulated induction times: solid red circles for bubble vs PP11-air and solid blue squares for bubble vs PP11 - water. In each case the symbol is for rupture thickness of $100 \mathrm{~nm}$ with upper and lower error bars giving $150 \mathrm{~nm}$ and $50 \mathrm{~nm}$ film rupture thicknesses, marked by horizontal dashed lines in (a). 


\section{CONCLUSIONS}

By using bubbles and water droplets of diameter $100 \mu \mathrm{m}$ to $1400 \mu \mathrm{m}$ in the fluorocarbon liquid PP11, we can study the effects of mobile or immobile hydrodynamic boundary conditions on the terminal velocity in a practical range up to $10 \mathrm{~cm} / \mathrm{s}$ that corresponds to a Reynolds number of up to 10. Bubbles in PP11 behaved as having a fully mobile interface whereas water droplets in PP11 had an immobile interface, like that of a solid. The experimental results are in excellent agreement with existing models.

The coalescence induction time of bubbles at a PP11-air and PP11-water interface differ by 2 to 3 orders of magnitude. The short inductions times for coalescence at the PP11-air interface is less than $1 \mathrm{~s}$ for the range of bubble size considered. This is attributed to the fact that both PP11-air interfaces are fully mobile, thus offering little resistance to the thinning of the PP11 film. The results are very reproducible suggesting that PP11-air interfaces have stable and consistent properties. The induction time increases as the square of the bubble size.

The coalescence induction times of bubbles at the immobile PP11-water interfaces are much longer and exhibit considerable scatter over a decade of values. Since electrostatic effects that may arise at the PP11-water interface have been suppressed by the addition of $0.5 \mathrm{M} \mathrm{NaCl}$ at pH 3 in the aqueous phase, and PP11 and water have almost identical refractive index in the visible light, the van der Waals forces also expected to be very small. The observed induction times correspond to rupture of the PP11 film between water and air at a thickness of around 100 $\mathrm{nm} \pm 50 \mathrm{~nm}$. The absence of obvious surface forces that can trigger film rupture and the large scatter in the observed induction times, suggest that surface contaminants that originated in the aqueous phase are implicated in the film rupture process. 


\section{SUPPORTING INFORMATION AVAILABLE}

Video 1. This combined video compares the free rise of a $196 \mu \mathrm{m}$ diameter bubble in PP11 toward the free PP11-air surface (left) or PP11-water interface (right). The video is shot at 1000 fps and played at $30 \mathrm{fps}$

Video 2. This video compares the collision of a $520 \mu \mathrm{m}$ diameter bubble with a PP11-air surface (left) or a PP11-water interface (right). The video is shot at $1000 \mathrm{fps}$ and played at $30 \mathrm{fps}$;

Video 3. This video compares the collision of a $950 \mu \mathrm{m}$ bubble with a PP11- air surface (left) or a PP11-water interface (right). The video is shot at $1000 \mathrm{fps}$ and played at $30 \mathrm{fps}$;

Video 4. This video shows the collision and subsequent coalescence of a $415 \mu \mathrm{m}$ diameter bubble with the PP11-water interface. The video is shot at $50 \mathrm{fps}$ and played at $30 \mathrm{fps}$.

This material is available free of charge via the Internet at http://pubs.acs.org.

\section{ACKNOWLEGMENTS}

This work was supported by the King Abdullah University of Science and Technology (KAUST). D. Y. C. C. was supported by the Australian Research Council through a Discovery Project Grant No. DP170100376. We acknowledge Mr. Yuansi Tian for assistance in some of the experiments. 


\section{REFERENCES}

1. Chan, D. Y. C.; Manica, R.; Klaseboer, E. Film drainage and coalescence between deformable drops and bubbles. Soft Matter, 2011, 7, 2235-2264.

2. Sanfeld, A.; Steinchen, A. Emulsions stability, from dilute to dense emulsions Role of drops deformation. Adv. Colloid Interface Sci., 2008, 140, 1-65.

3. Ivanov, I.; Dimitrov, D. Thin Liquid Films; Marcel Dekker: New York, 1988.

4. Lauga, E.; Stone, H. A. Effective slip in pressure-driven Stokes flow. J. Fluid Mech. 2003, 489, 55-77.

5. Yoon, Y.; Baldessari, F.; Ceniceros, H. D.; Leal, L. G. Coalescence of two equalsized deformable drops in an axisymmetric flow. Phys. Fluids, 2007, 19, 102102.

6. A. K. Chesters and G. Hofman, Bubble coalescence in pure liquids. Appl. Sci. Res. 1982, 38, 353-361.

7. Parkinson, L.; Sedev R.; Fornasiero, D.; Ralston, J. The terminal rise velocity of 10-100 $\mu \mathrm{m}$ diameter bubbles in water. J. Colloid Interface Sci. 2008, 322, 168172.

8. Kelsall, G. H.; Tang, S.; Smith, A. L.; Yurdakul, S. Measurement of rise and electrophoretic velocities of gas bubbles J. Chem. Soc. Faraday Trans. 1996, 92, $3879-3885$. 
9. Duineveld, P. C. The rise velocity and shape of bubbles in pure water at high Reynolds number, J. Fluid Mech. 1995, 292, 325-332.

10. Kosior, D.; Zawala, J.; Krasowska, M.; Malysa, K. Influence of n-octanol and $\alpha$ terpineol on thin film stability and bubble attachment to hydrophobic surface. Phys. Chem. Chem. Phys. 2013, 15, 2586-2595.

11. Malysa, K.; Krasowska, M.; Krzan, M. Influence of surface active substances on bubble motion and collision with various interfaces. Adv. Colloid Interface Sci. 2005, 114-115, 205-225.

12. Zawala, J.; Krasowska, M.; Dabros, T.; Malysa, K.; Influence of bubble kinetic energy on its bouncing during collisions with various interfaces. Can. J. Chem. Eng. 2007, 85, 669-678.

13. Zawala, J.; Malysa, K. Influence of the impact velocity and size of the film formed on bubble coalescence time at water surface. Langmuir 2011, 27, 2250-2257.

14. Zawala, J.; Dorbolo, S.; Terwagne, D.; Vandewalle, N.; Malysa, K. Bouncing bubble on a liquid/gas interface resting or vibrating. Soft Matter 2011, 7, 67196726.

15. Kosior, D.; Zawala, J.; Malysa, K. Influence of n-octanol on the bubble impact velocity, bouncing and the three phase contact formation at hydrophobic solid surfaces. Colloids Surf. A 2014, 441, 788-795. 
16. Kosior, D.; Zawala, J.; Todorov, R.; Exerowa D.; Malysa, K. Bubble bouncing and stability of liquid films formed under dynamic and static conditions from noctanol solutions. Colloids and Surfaces A 2014, 460, 391-400.

17. Hendrix, M. H. W.; Manica, R.; Klaseboer, E.; Chan D. Y. C.; Ohl, C.-D. Spatiotemporal evolution of thin liquid films during impact of water bubbles on glass on a micrometer to nanometer scale. Phys. Rev. Lett. 2012, 108, 247803.

18. Manica, R.; Hendrix, M. H. W.; Gupta, R.; Klaseboer, E.; Ohl C.-D.; Chan, D. Y. C. Effects of hydrodynamic film boundary conditions on bubble-wall impact. Soft Matter 2013, 9, 9755-9758.

19. Parkinson, L.; Ralston J. The interaction between a very small rising bubble and a hydrophilic titania surface. J. Phys. Chem. C 2010, 114, 2273-2281.

20. Manica, R.; Parkinson, L.; Ralston J.; Chan, D.Y.C. Interpreting the dynamic interaction between a very small rising bubble and a hydrophilic titania surface. $J$. Phys. Chem. C 2010, 114,1942-1946.

21. Manica, R.; Klaseboer, E.; Chan, D. Y. C. The impact and bounce of air bubbles at a flat fluid interface. Soft Matter 2016, 12, 3271-3282.

22. Vakarelski, I. U.; Manica, R.; Tang, X.S.; O’Shea, S. J.; Stevens, G. W.; Grieser, F.; Dagastine, R. R.; Chan, D. Y. C. Dynamic interactions between microbubbles in water. Proc. Natl. Acad. Sci. U. S. A. 2010, 107, 11177-11182. 
23. Tabor, R. F.; Wu, C. Grieser, F. Dagastine, R. R.; Chan, D. Y. C. Measurement of the hydrophobic force in a soft matter system. J. Phys. Chem. Lett. 2013, 4, 38723877.

24. Shi, C.; Cui, X.; Xie, L.; Liu, Q. X.; Chan, D. Y. C.; Israelachvili J. N.; Zeng, H. B. Measuring forces and spatiotemporal evolution of thin water films between an air bubble and solid surfaces of different hydrophobicity. ACS Nano 2015, 9, 95104.

25. Pushkarova R. A.; Horn, R. G. Bubble-solid interactions in water and electrolyte solutions. Langmuir 2008, 24, 8726-8734.

26. Zhang, X.; Tchoukov, P.; Manica, R.; Wang, L.; Liu Q.; Xu, Z. Simultaneous measurement of dynamic force and spatial thin film thickness between deformable and solid surfaces by integrated thin liquid film force apparatus. Soft Matter 2016, $12,9105-9114$.

27. Manica, R.; Connor, J. N.; Carnie, S. L.; Horn R. G.; Chan, D. Y. C. Dynamics of interactions involving deformable drops: Hydrodynamic dimpling under attractive and repulsive electrical double layer interactions. Langmuir 2007, 23, 626-637.

28. Yoon, Y.; Borrell, M.; Park, C. C.; Leal, L. G. Viscosity ratio effects on the coalescence of two equal-sized drops in a two-dimensional linear flow. J. Fluid Mech., 2005, 525, 355-379.

29. Kočárková, H.; Rouyer F.; Pigeonneau, F. Film drainage of viscous liquid on top of bare bubble: Influence of the bond number. Phys. Fluids 2013, 25, 022105. 
30. Pigeonneau F.; Sellier, A. Low-Reynolds-number gravity-driven migration and deformation of bubbles near a free surface. Phys. Fluids 2011, 23, 092102.

31. Nguyen P. T.; Nguyen, A. V. Drainage, rupture, and lifetime of deionized water films: Effect of dissolved gases? Langmuir 2010, 26, 8061-8074.

32. Yaminsky, V. V.; Ohnishi, S.; Vogler, E. A.; Horn, R. G. Stability of aqueous films between bubbles. Part 1. The effect of speed on bubble coalescence in purified water and simple electrolyte solutions. Langmuir 2010, 26, 8061-8074

33. Yaminsky, V. V.; Ohnishi, S.; Vogler, E. A.; Horn, R. G. Stability of aqueous films between bubbles. Part 2 Effects of Trace Impurities and Evaporation Langmuir 2010, 26, 8075-8080.

34. Vakarelski, I. U.; Klaseboer, E.; Jetly, A.; Mansoor, M. M.; Aguirre-Pablo, A. A.; Chan D. Y. C.; Thoroddsen S. T. Self-determined shapes and velocities of giant near-zero drag gas cavities. Sci. Adv. 2017, 3, e1701558.

35. Vinogradova, O. I. Slippage of water over hydrophobic surfaces. Int. J. Miner. Process. 1999, 56. 31-60.

36. Li, E. Q.; Vakarelski, I. U.; Chan D. Y. C.; Thoroddsen, S. T. Stabilization of thin liquid films by repulsive van der Waals force. Langmuir 2014, 30, 5162-5169.

37. Vakarelski, I. U.; Berry, J. D.; Chan D. Y. C.; Thoroddsen, S. T. Leidenfrost vapor layers reduce drag without the crisis in high viscosity liquids. Phys. Rev. Lett. 2016, 117,114503 . 
38. Marinova, K. G.; Alargova, R. G.; Denkov, N. D.; Velev, O. D.; Petsev, D. N.; Ivanov, I. B.; Borwankar, R. P. Charging of oil-water interfaces due to spontaneous adsorption of hydroxyl ions. Langmuir 1996, 12, 2045-2051.

39. Tang, Y.; Yan, J.; Zhou, X.; Fu, Y.; Mao, B. An STM study on nonionic fluorosurfactant Zonyl FSN self-assembly on Au(111): Large domains, few defects, and good stability. Langmuir 2008, 24, 13245-13249.

40. Scheludko, A.; Exerowa, D. Device for interferometric measurement of the thickness of microscopic foam films. Comm. Dept. Chem. Bull. Acad. Sci. 1959, 7, 123-132.

41. Scheludko, A. Thin liquid films. Adv. Colloid Interface Sci. 1967, 1, 391-464.

42. Hadamard, Mouvement permanent lent d'une sphere liquide et visqueuse dans un liquide visqueux. J. S.; C. R. Acad. Sci. 1911, 152, 1735-1738.

43. Rybczynski, W. Über die fortschreitende Bewegung einer flüssigen Kugel in einem zähen Medium. Bull. Acad. Sci. Cracow Ser. A 1911, 40-46.

44. Levich, V. G. Physicochemical Hydrodynamics, Prentice-Hall International Series in the Physical and Engineering Sciences, Prentice-Hall, Englewood Cliffs, 1962, p. 700 .

45. Mei, R.; Klausner, J. F.; Lawrence, C. J. A note on the history force on a spherical bubble at finite Reynolds number. Phys. Fluids 1994, 6, 418-420.

46. Schiller, L.; Naumann, A. Z. Über die grundlegenden Berechnungen bei der Schwerkraftaufbereitung. Z. Ver. Deutsch. Ing. 1933, 77, 318-320. 
47. Moore, D.W. The velocity of rise of distorted gas bubbles in a liquid of small viscosity. J. Fluid Mech. 1965, 23, 749-766.

48. Bart, E. The slow unsteady settling of a fluid sphere toward a flat fluid interface Chem. Eng. Sci. 1968, 23, 193-210.

49. Katsir Y.; Marmur, A. Rate of bubble coalescence following dynamic approach: collectivity-induced specificity of ionic effect. Langmuir 2014, 30, 13823-13830.

50. Langevin, D. Bubble coalescence in pure liquids and in surfactant solutions, Curr. Opin. Colloid Interface Sci. 2015, 20, 92-97.

51. Israelachvili, J. N. Intermolecular and Surface Forces, Academic Press Ltd., San Diego, $3^{\text {rd }}$ ed., 2011 , p. 263.

52. Yiantsios S. G.; Davis, R. H. Close approach and deformation of two viscous drops due to gravity and van der Waals forces. J. Colloid Interface Sci. 1991, 144, 412-433. 


\section{FOR TABLE OF CONTENTS USE ONLY}

\section{Coalescence dynamics of mobile and immobile fluid interfaces}

Ivan U. Vakarelski, Rogerio Manica, Er Qiang Li, Elka S. Basheva, Derek Y.C. Chan and Sigurdur T. Thoroddsen

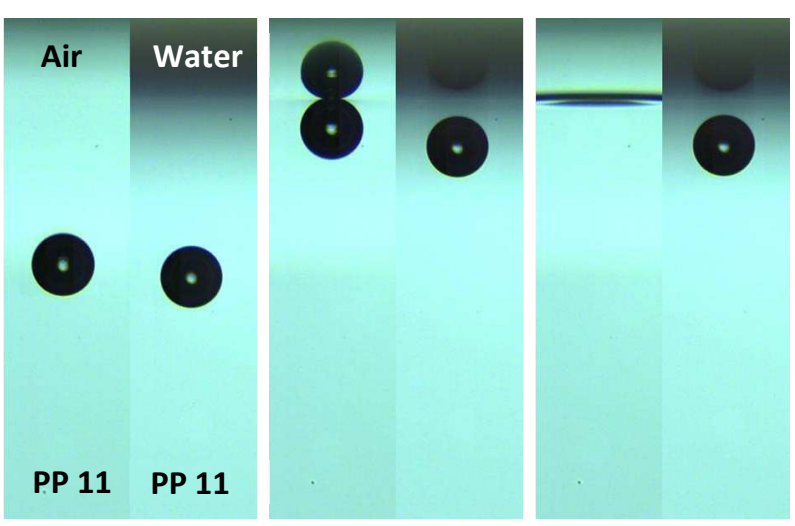

ACS Paragon Plus Environment 